\title{
Magneto-optical transport properties of monolayer $\mathrm{MoS}_{2}$ on polar substrates
}

\author{
Chuong V. Nguyen, ${ }^{1,2}$ Nguyen N. Hieu, ${ }^{1}$ Nikolai A. Poklonski, ${ }^{3}$ Victor V. Ilyasov, ${ }^{4}$ Le Dinh, ${ }^{5}$ \\ Tran C. Phong, ${ }^{5,6}$ Luong V. Tung, ${ }^{7}$ and Huynh V. Phuc ${ }^{7, *}$ \\ ${ }^{1}$ Institute of Research and Development, Duy Tan University, Da Nang 550000, Vietnam \\ ${ }^{2}$ Department of Materials Science and Engineering, Le Quy Don Technical University, Hanoi 100000, Vietnam \\ ${ }^{3}$ Physics Department, Belarusian State University, Minsk 220030, Belarus \\ ${ }^{4}$ Department of Physics, Don State Technical University, Rostov on Don 344000, Russia \\ ${ }^{5}$ Center for Theoretical and Computational Physics, Hue University's College of Education, Hue 530000, Vietnam \\ ${ }^{6}$ Vietnam Institute of Educational Sciences, 101 Tran Hung Dao, Hanoi 100000, Vietnam \\ ${ }^{7}$ Division of Theoretical Physics, Dong Thap University, Dong Thap 870000, Vietnam
}

(Received 15 May 2017; revised manuscript received 21 July 2017; published 8 September 2017)

\begin{abstract}
We theoretically study the magneto-optical transport properties of monolayer molybdenum disulfide $\left(\mathrm{MoS}_{2}\right)$ on polar substrates in the presence of a perpendicular magnetic field. The magneto-optical absorption coefficient (MOAC) is investigated as a function of the incident photon energy when carriers are scattered by three different types of phonons: the intrinsic $\mathrm{MoS}_{2}$ acoustic, optical phonons, and the surface optical (SO) phonons induced by polar substrates. Among the substrates considered, the largest magnitude of MOAC and full-width at half maximum (FWHM) are observed for a $\mathrm{SiO}_{2}$ substrate over the entire temperature and magnetic field range considered due to its strongest electron-SO phonon scattering, while an h-BN substrate displays the lowest one. The piezoelectric (PE) coupling to the transverse (TA) phonon is shown to dominate the MOAC and FWHM due to intrinsic acoustic phonon scattering. Meanwhile, these properties for intrinsic optical phonons are dominated by zero-order deformation potential (DP) couplings and the Fröhlich interaction. The dependence of the MOAC and FWHM on temperature, magnetic field, and the effective $\mathrm{MoS}_{2}$-substrate distance is also examined. The present results for monolayer $\mathrm{MoS}_{2}$ are compared with those in conventional two-dimensional systems as well as in graphene. Our results show that SO phonons play a crucial role at high temperature depending on the substrates and have a non-negligible effect on the magneto-optical transport properties of monolayer $\mathrm{MoS}_{2}$, which could be further experimentally and theoretically investigated in the future.
\end{abstract}

DOI: 10.1103/PhysRevB.96.125411

\section{INTRODUCTION}

In parallel with the rise of graphene and the discovery of its outstanding electronic properties as well as the use of this material in applications [1-3], the focus has been shifting to the other two-dimensional (2D) transition-metal dichalcogenides with promising electronic properties and potential applications [4-6]. The reason for this trend originates from the zero band gap of graphene, a property essential for electronic applications $[7,8]$. Although having the honeycomb structure of graphene, molybdenum disulfide $\left(\mathrm{MoS}_{2}\right)$ is a direct-gap semiconductor with a strong spin-orbit coupling (SOC), a large natural band gap, and massive Dirac fermions [9-12]. It has been demonstrated that monolayer $\mathrm{MoS}_{2}$ has a large carrier mobility [13], high thermal stability, good compatibility with standard semiconductor manufacturing [5], and high current carrying capacity [14]. These properties make $\mathrm{MoS}_{2}$ a promising alternative to graphene and have made it a candidate for a wide range of applications such as field-effect transistors $[5,15]$, valleytronics devices $[6,16-18]$, spintronic devices [19], and photonics [20].

Monolayer $\mathrm{MoS}_{2}$ is composed of a single layer of molybdenum (Mo) atoms interleaved between two layers of sulfur (S) atoms in a trigonal prismatic structure [6,21]. Its electronic and band structure can be obtained from the $\mathbf{k} \cdot \mathbf{p}$ theory framework [9], the continuum theory [22], or from the more

\footnotetext{
*hvphuc@dthu.edu.vn
}

complicated multiband $\mathbf{k} \cdot \mathbf{p}$ model [23]. $\mathrm{MoS}_{2}$ has been demonstrated to be very sensitive to external conditions, such as electric field [24] and strain engineering [25,26], leading to a crossover from an indirect-band-gap semiconductor at multilayer to a direct-band-gap semiconductor at monolayer [9]. For its interesting and important electronic and optical properties, $\mathrm{MoS}_{2}$ has been investigated intensively in recent years. Having calculated the longitudinal and spin-valley Hall optical conductivity in monolayer $\mathrm{MoS}_{2}, \mathrm{Li}$ and Carbotte [27] observed the optical peaks due to the interband transitions from the valence to the conduction bands located in the visible range. He et al. [28] investigated the magnetic properties of nonmetal atoms absorbed monolayer $\mathrm{MoS}_{2}$ by first-principles calculations. The spin- and valley-dependent magneto-optical properties [29], the quantum magnetotransport $[8,12,30]$, the spin- and valley-polarized transport [31], and the effect of doping and strain modulations on electron transport [32], as well as the thermal conductivity and phonon linewidths [33] have been investigated in this interesting system.

Besides leading to the renormalization in band gap and introducing the extra peaks in the magneto-optical conductivity [34-36], the electron-phonon interaction plays also a salient role in the investigation into transport properties of monolayer $\mathrm{MoS}_{2}$ such as: phonon limited mobility [7,37], hot-electron cooling [38], free-carrier optical absorption [39], thermoelectric transport coefficients [40], electrical transport properties [41], and magneto-optical transitions [42]. Recently, using a density-functional-based approach, Kaasbjerg et al. [7] have calculated the electron-phonon couplings in monolayer 
$\mathrm{MoS}_{2}$ for acoustic and optical deformation potentials as well as the Fröhlich interaction. They have studied the phonon-limited mobility for both transverse acoustic (TA) and longitudinal acoustic (LA) modes. In the case of optical phonon scattering, they have dealt with both couplings to zero and first order in $q$ corresponding to the interaction with the homopolar mode (HP) and the polar transverse optical (TO) phonon, respectively. Meanwhile the Fröhlich interaction corresponds to the polar longitudinal optical (LO) phonon. Besides, the influence of the substrates on the monolayer material plays a crucial role in determining the basic properties of the monolayer $\mathrm{MoS}_{2} /$ substrates heterostructure [43]. The strong coupling between the carriers of monolayer materials and the remote optical-phonon modes resulting from the polar substrates lead to extended surface-optical (SO) phonon scattering [42]. Therefore, the carrier-SO phonon scattering in graphene [15,44,45] and black phosphorus [46] on polar substrates have been reported with the optimal distances $d$ between materials and substrates of $0.35 \mathrm{~nm}$ [44], from 0.2 to $0.8 \mathrm{~nm}[15]$, or even up to $1 \mathrm{~nm}[45,46]$. However, carrierphonon scattering, especially carrier-SO phonon scattering, in $\mathrm{MoS}_{2}$ on polar substrates has not been studied very much so far.

Magneto-optics are known to be the most important tools to measure band structure of metals and semiconductors experimentally [47]. The magneto-optical properties of graphene [44], of topological insulators [48,49], $\mathrm{WSe}_{2}$ [50], and of Weyl semimetals [51] have been investigated. These useful properties have also been studied in other monolayer materials such as silicene [52,53], phosphorene $[54,55]$, and $\mathrm{WSe}_{2}$ [56] using the Kubo formula. We realize that though many efforts have been made to investigate the magneto-optical properties of $\mathrm{MoS}_{2}$ [8,29,57], a detailed investigation of the effect of different types of phonons as well as the effect of the two-photon absorption process on the magneto-optical transport properties of monolayer $\mathrm{MoS}_{2}$ on polar substrates has not received enough attention. Therefore, studying the magneto-optical transport properties is timely and expected to increase our understanding of this interesting material.

In this work, we study the magneto-optical transport properties of monolayer $\mathrm{MoS}_{2}$ on polar substrates in a perpendicular magnetic field via evaluating MOAC and FWHM. The results are calculated in a large range of temperatures and magnetic fields including the effect of different types of phonons as well as the effect of the two-photon absorption process. In Sec. II we present the basics of the model. In Sec. III we show magneto-optical absorption coefficients. The numerical results and discussion are described in Sec. IV. Finally, we show our summary and conclusions in Sec. V.

\section{BASIC FORMULATION}

In the presence of a uniform static perpendicular magnetic field $\mathbf{B}=(0,0, B)$ applied along the $z$ direction, the Hamiltonian of monolayer $\mathrm{MoS}_{2}$ without Zeeman terms can be written as

$$
\mathcal{H}=\mathcal{H}_{e}+\mathcal{H}_{\text {ph }}+\mathcal{H}_{\text {int }}
$$

Here the electronic single-particle Hamiltonian is defined as follows $[8,9,58]$ :

$$
\mathcal{H}_{e}=\operatorname{at}\left(\tau k_{x} \sigma_{x}+k_{y} \sigma_{y}\right)+(\bar{\Delta}-s \tau \bar{\lambda}) \sigma_{z}+s \tau \bar{\lambda},
$$

where $a$ is the lattice constant, $t$ is the hopping integral, $\bar{\Delta}=$ $\Delta / 2, \bar{\lambda}=\lambda / 2$ with $\Delta$ and $\lambda$ being, respectively, the energy gap and SOC parameter, the valley index $\tau= \pm 1$ refers to $K$ and $K^{\prime}$ valleys, the spin index $s= \pm 1$ stands for spin up and down, $\mathbf{k}=\left(k_{x}, k_{y}\right)$ is the momentum of the carrier, and $\sigma_{i}(i=x, y, z)$ are the Pauli matrices for the two basic functions. Note that the Zeeman effects are also important [12,50]. However, the main purpose of this work is to study the magneto-optical transport properties of monolayer $\mathrm{MoS}_{2}$ due to the effect of different types of phonons as well as the effect of the twophoton absorption process. Therefore, we neglect the Zeeman effects in the present study. The energy spectrum $E_{\alpha}$ in the electronic states $|\alpha\rangle=|n, s, \eta, \tau\rangle$ is given as follows [8]:

$$
E_{\alpha}=E_{n s \eta \tau}=s \tau \bar{\lambda}+\eta \sqrt{\bar{\Delta}_{s \tau}^{2}+n\left(\hbar \omega_{c}\right)^{2}}
$$

for $n \geqslant 1$, with $n$ being an integer denoting the Landau level (LL) index, $\bar{\Delta}_{s \tau}=\bar{\Delta}-s \tau \bar{\lambda}$, the band index $\eta= \pm 1$ is for the conduction and valence bands, and $\hbar \omega_{c}=a t \sqrt{2} / a_{c}$ is the cyclotron energy with $a_{c}=(\hbar / e B)^{1 / 2}$ being the magnetic length. For $n=0$, the eigenvalue is

$$
E_{0 s \tau}=-\tau(\bar{\Delta}-s \bar{\lambda})+s \bar{\lambda} .
$$

Noting that $\hbar \omega_{c} \leqslant \bar{\Delta}_{s \tau}$ and expanding the square root in Eq. (3), we obtain a simpler expression for the eigenvalues

$$
E_{n s \eta \tau} \approx(1-\eta) s \tau \bar{\lambda}+\eta \bar{\Delta}+n \eta \frac{\hbar^{2} \omega_{c}^{2}}{2 \bar{\Delta}_{s \tau}},
$$

which shows a regularly linear dependence of the LLs on $n$ and $B$. Since $\bar{\Delta} \geqslant s \tau \bar{\lambda}$ the last term in Eq. (5) is equal to $n \eta\left(\hbar^{2} \omega_{c}^{2} / 2 \bar{\Delta}\right)(1+s \tau \bar{\lambda} / \bar{\Delta})$ giving a spin splitting $E_{(s=1)}-E_{(s=-1)}=n \tau \bar{\lambda}\left(\hbar \omega_{c} / \bar{\Delta}\right)^{2}$ in the conduction band and $4 \tau \bar{\lambda}-n \tau \bar{\lambda}\left(\hbar \omega_{c} / \bar{\Delta}\right)^{2}$ in the valence band. Because $\hbar \omega_{c} \leqslant \bar{\Delta}$, the term $n \tau \bar{\lambda}\left(\hbar \omega_{c} / \bar{\Delta}\right)^{2}$ is important in the conduction band but negligible in the valence band.

The eigenfunctions for the $K$ valley $(\tau=+1)$ are

$$
\Phi_{n, s}^{\eta,+1}\left(\mathbf{r}, k_{y}\right)=\frac{e^{i k_{y} y}}{\sqrt{L_{n, s}^{\eta,+1}}}\left(\begin{array}{c}
\Lambda_{n, s}^{\eta,+1} \phi_{n-1}\left(x-x_{0}\right) \\
\phi_{n}\left(x-x_{0}\right)
\end{array}\right) .
$$

Here $\phi_{n}\left(x-x_{0}\right)$ represents the harmonic oscillator eigenfunction, centered at $x_{0}=a_{c}^{2} k_{y}$. The factor $L_{n, s}^{\eta, \tau}$ is

$$
L_{n, s}^{\eta, \tau}=\left(\Lambda_{n, s}^{\eta, \tau}\right)^{2}+1,
$$

with $\Lambda_{n, s}^{\eta, \tau}=\sqrt{n} \hbar \omega_{c}\left[(1-\eta \tau) \bar{\Delta}_{s \tau}-n \eta \tau \hbar^{2} \omega_{c}^{2} / 2 \bar{\Delta}_{s \tau}\right]^{-1}$. The eigenfunctions for the $K^{\prime}$ valley $(\tau=-1)$ are obtained from Eq. (6) by exchanging $\phi_{n}\left(x-x_{0}\right)$ and $\phi_{n-1}\left(x-x_{0}\right)$.

The second term of Eq. (1) is the phononic Hamiltonian including different phonon branches (labeled as $v$ ). These phonons are characterized by their dispersion $\omega_{\mathbf{q}, v}$ and momentum q, and their corresponding Hamiltonian

$$
\mathcal{H}_{\mathrm{ph}}=\sum_{\mathbf{q}, v} \hbar \omega_{\mathbf{q}, v} b_{\mathbf{q}, v}^{\dagger} b_{\mathbf{q}, v},
$$




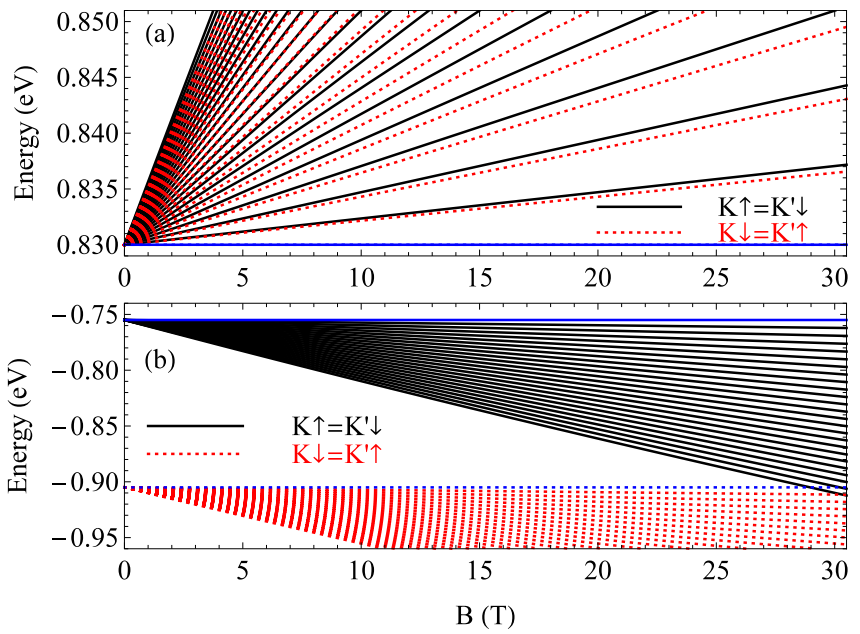

FIG. 1. Energy spectrum of monolayer $\mathrm{MoS}_{2}$ as a function of magnetic field $B$. The top (a) and bottom (b) panels correspond to the conduction and valence bands, respectively.

where $b_{\mathbf{q}, v}^{\dagger}$ and $b_{\mathbf{q}, v}$ are the creation and annihilation operators of a phonon, respectively. The last term in Eq. (1) is the carrierphonon interaction part of the Hamiltonian, given by [8]

$$
\mathcal{H}_{\text {int }}=\sum_{\mathbf{q}, v} \sum_{\alpha, \alpha^{\prime}} \mathrm{g}_{\mathbf{q}, v} J_{\alpha, \alpha^{\prime}}(q) e^{i \mathbf{q} \cdot \mathbf{r}} a_{\alpha}^{\dagger} a_{\alpha^{\prime}} \phi_{\mathbf{q}, v},
$$

where $\mathrm{g}_{\mathbf{q}, v}$ is the carrier-phonon coupling matrix element, which depends on the phonon branch as well as on the coupling mechanism. $\phi_{\mathbf{q}, v}=\left(b_{-\mathbf{q}, v}^{\dagger}+b_{\mathbf{q}, v}\right)$ is the phonon field operator. The form factor for the intravalley case $\left(\tau^{\prime}=\tau\right)$ is given as follows:

$$
\begin{aligned}
\left|J_{\alpha, \alpha^{\prime}}(q)\right|^{2}= & \frac{\delta_{s s^{\prime}}}{L_{n, s}^{\eta, \tau} L_{n^{\prime}, s^{\prime}}^{\eta^{\prime}, \tau}} u^{j} e^{-u} \frac{m !}{(m+j) !} \\
& \times\left[\Lambda_{n, s}^{\eta, \tau} \Lambda_{n^{\prime}, s^{\prime}}^{\eta^{\prime}, \tau} \sqrt{\frac{m+j}{m}} L_{m-1}^{j}(u)+L_{m}^{j}(u)\right]^{2},
\end{aligned}
$$

where $u=a_{c}^{2} q^{2} / 2, m=\min \left(n, n^{\prime}\right), j=\left|n^{\prime}-n\right|$, and $L_{m}^{j}(u)$ are the associated Laguerre polynomials.

In Fig. 1 we present the eigenvalues given by Eqs. (4) and (5) as functions of the magnetic field $B$. The top and bottom panels are for the conduction and valence bands, respectively. The blue lines are for the zero level $(n=0)$. It can be noted that the zero level for the $K$ valley is in the valence band and depends on the spin index. Meanwhile for the $K^{\prime}$ valley, it lies in the conduction band and does not depend on the spin index. This can be observed from Eq. (4): for the $K^{\prime}$ valley $(\tau=-1)$ Eq. (4) reduces to $E_{0 s-1}=\bar{\Delta}$, which is independent from the $s$ index. Moreover, in contrast to graphene $[59,60]$ or silicene [52] where the energies of the LLs depend on $\sqrt{B}$, here the energies of the LLs in $\mathrm{MoS}_{2}$ increases linearly with $B$. This can be explicitly observed from Eq. (5). In the conduction band, the spin splitting is equal to $n \tau \bar{\lambda}\left(\hbar \omega_{c} / \bar{\Delta}\right)^{2}$ which depends linearly on the LL index $n$ and magnetic field $B$. Meanwhile, the spin splitting is $4 \tau \bar{\lambda}-n \tau \bar{\lambda}\left(\hbar \omega_{c} / \bar{\Delta}\right)^{2}$ in the valence band, with a value $|4 \tau \bar{\lambda}|=150 \mathrm{meV}$ at $B=0$, in complete agreement with previous works $[9,12]$. As can be seen from Fig. 1, the energies of the spin up (down) LLs at the $K$ and $K^{\prime}$ valleys are opposite. This feature is in contrast to the previous work [12] where the Zeeman effect is included, but agrees with the zero magnetic field limit which results from time-reversal symmetry [9].

To obtain the optical absorption intensity in the presence of the magnetic field, we calculate the magneto-optical absorption coefficient (MOAC). When an electromagnetic field with energy $\hbar \Omega$ is applied to the system, the MOAC due to photon absorption with simultaneous absorption and emission of a phonon of the $v$ branch is given as [61]

$$
K^{v}(\Omega)=\frac{1}{V(I / \hbar \Omega)} \sum_{\alpha, \alpha^{\prime}} \mathcal{W}_{\alpha, \alpha^{\prime}}^{ \pm, v} f_{\alpha}\left(1-f_{\alpha^{\prime}}\right),
$$

where $V$ is the volume of the system, and $I / \hbar \Omega$ is the injected number of photons per unit area per second with the optical intensity $I=n_{r} c \varepsilon_{0} \Omega^{2} A_{0}^{2} / 2$. Here $n_{r}$ is the refractive index of the material, $c$ is the speed of light in free space, $\varepsilon_{0}$ is the permittivity of vacuum, and $A_{0}$ is the amplitude of the vector potential for the electromagnetic field. In Eq. (11), $f_{\alpha}$ and $f_{\alpha^{\prime}}$ are the carrier distribution functions in the initial and final states, which should be obtained with Fermi-Dirac statistics because of the high density of states in the $K$ and $K^{\prime}$ valleys of the conduction band in monolayer $\mathrm{MoS}_{2}$ [7]. The sums run over all quantum numbers of the states $|\alpha\rangle \equiv|n, s, \eta, \tau\rangle$ and $\left|\alpha^{\prime}\right\rangle \equiv\left|n^{\prime}, s^{\prime}, \eta^{\prime}, \tau^{\prime}\right\rangle$ provided $\alpha^{\prime} \neq \alpha$. The transition matrix element per unit area for carrier-photon-phonon interaction of the 2D carrier [45,62], including the $\ell$-photon absorption process [63,64], is given by Born's second-order golden rule [65] and takes into account the properties of the $\mathrm{MoS}_{2}$ eigenfunctions

$$
\begin{aligned}
\mathcal{W}_{\alpha, \alpha^{\prime}}^{ \pm, v}= & \frac{2 \pi}{\hbar} \sum_{\mathbf{q}} \sum_{\ell=1}^{\infty}\left(\left|\mathcal{M}_{\alpha, \alpha^{\prime}}^{ \pm, v}\right|^{2}\left|\mathcal{M}_{\alpha, \alpha^{\prime}}^{\mathrm{rad}}\right|^{2} / \hbar^{2} \Omega^{2}\right) \\
& \times \frac{\left(\alpha_{0} q\right)^{2 \ell}}{(\ell !)^{2} 2^{2 \ell}} \delta\left(E_{\alpha^{\prime}}-E_{\alpha}-\ell \hbar \Omega \pm \hbar \omega_{\mathbf{q}, v}\right),
\end{aligned}
$$

where the upper $(+)$ and lower $(-)$ signs refer to the emission and absorption processes of phonons, respectively, and $\alpha_{0}$ is the dressing parameter. The carrier-phonon matrix element $\mathcal{M}_{\alpha, \alpha,}^{ \pm, v}$ is given by

$$
\left|\mathcal{M}_{\alpha, \alpha^{\prime}}^{ \pm, v}\right|^{2}=\left|\mathrm{g}_{\mathbf{q}, v}\right|^{2} \cos ^{2}\left(\frac{\theta}{2}\right)\left|J_{\alpha, \alpha^{\prime}}(q)\right|^{2} N_{\mathbf{q}, v}^{ \pm},
$$

where $N_{\mathbf{q}, v}^{ \pm}=N_{\mathbf{q}, v}+1 / 2 \pm 1 / 2$ for the Bose factor $N_{\mathbf{q}, v}$ which presents the number of $(\mathbf{q}, v)$ phonons, and $\theta$ is the scattering angle. The steady-state matrix element for carrierphoton interaction is [61]

$$
\left|\mathcal{M}_{\alpha, \alpha^{\prime}}^{\mathrm{rad}}\right|^{2}=\frac{\Omega^{2} A_{0}^{2}}{4}\left|\mathbf{e} \cdot e \mathcal{B}_{\alpha \alpha^{\prime}}\right|^{2},
$$

where $\mathbf{e}$ is the polarized vector of the electromagnetic field, and $e \mathcal{B}_{\alpha \alpha^{\prime}}=e\left\langle\alpha^{\prime}|\mathbf{r}| \alpha\right\rangle$ is the dipole moment with $e$ being the absolute value of the electron charge.

\section{MAGNETO-OPTICAL ABSORPTION COEFFICIENTS}

By means of the eigenfunctions in Eq. (6), and assuming the electromagnetic field to be linearly polarized transverse to 
the magnetic field, the steady-state matrix element for carrierphoton interaction in Eq. (14) is calculated as follows for the intravalley case:

$$
\mathcal{M}_{\alpha, \alpha^{\prime}}^{\mathrm{rad}}=\frac{e \Omega A_{0}}{2} \mathcal{B}_{\alpha \alpha^{\prime}},
$$

where the matrix element of the position operator is

$$
\begin{aligned}
\mathcal{B}_{\alpha \alpha^{\prime}}= & \left(\Lambda_{n+1, s}^{\eta, \pm 1} \Lambda_{n^{\prime}+1, s^{\prime}}^{\eta^{\prime}, \pm 1}+1\right)\left[x_{0} \delta_{n^{\prime}, n}+\left(a_{c} / \sqrt{2}\right)\right. \\
& \left.\times\left(\sqrt{n} \delta_{n^{\prime}, n-1}+\sqrt{n+1} \delta_{n^{\prime}, n+1}\right)\right] \delta_{k_{y}^{\prime}, k_{y}} .
\end{aligned}
$$

From Eq. (16), we find that the transitions in monolayer $\mathrm{MoS}_{2}$ occur when the LL index changes as $\Delta n=0, \pm 1$, similar with that of dipole type transitions reported in graphene $[59,60]$, silicene [52], topological insulators [48,66], and phosphorene [55], but different from black phosphorus thin films [47].

The transition matrix element in Eq. (12) contains a contribution of the $\ell$-photon process; however, in this work, we only restrict ourselves to considering the two-photon process, i.e., $\ell=1,2$. In this case, the MOAC is found to be

$$
\begin{aligned}
K^{v}(\Omega)= & \mathcal{D}\left(\omega_{c}, \Omega\right) \sum_{\alpha, \alpha^{\prime}}\left|\mathcal{B}_{\alpha \alpha^{\prime}}\right|^{2} f_{\alpha}\left(1-f_{\alpha^{\prime}}\right) \int_{0}^{\infty} d q q^{3}\left|\mathrm{~g}_{\mathbf{q}, v}\right|^{2} \\
& \times\left|J_{\alpha, \alpha^{\prime}}(q)\right|^{2}\left\{N_{\mathbf{q}, v}^{-} \delta\left(\mathcal{X}_{1}^{-}\right)+N_{\mathbf{q}, v}^{+} \delta\left(\mathcal{X}_{1}^{+}\right)\right. \\
& \left.+\frac{\alpha_{0}^{2} q^{2}}{16}\left[N_{\mathbf{q}, v}^{-} \delta\left(\mathcal{X}_{2}^{-}\right)+N_{\mathbf{q}, v}^{+} \delta\left(\mathcal{X}_{2}^{+}\right)\right]\right\}
\end{aligned}
$$

where we have denoted

$$
\begin{gathered}
\mathcal{D}\left(\omega_{c}, \Omega\right)=\frac{g_{s} g_{v} \mathcal{A} e^{2} \alpha_{0}^{2}}{64 \pi n_{r} c \varepsilon_{0} \hbar^{2} \Omega a_{c}^{2}}, \\
\mathcal{X}_{\ell}^{ \pm}=E_{\alpha^{\prime}}-E_{\alpha}-\ell \hbar \Omega \pm \hbar \omega_{\mathbf{q}, v} \quad(\ell=1,2) .
\end{gathered}
$$

In Eq. (18), $g_{v}=2$ and $g_{s}=2$ are the valley and spin degeneracies, respectively, $\mathcal{A}$ is the area of the sample. The expression for MOAC shown in Eq. (17) can be evaluated for various kinds of carrier-phonon interactions.

When $\mathrm{MoS}_{2}$ is situated on a substrate, carriers couple with three different types of phonons: intrinsic $\mathrm{MoS}_{2}$ acoustic and optical phonons [7,37-39], and SO phonons induced by polar substrates [42,67]. In the following, the analytic expressions for MOAC are determined when the carriers are assumed to interact with these three different types of phonons.

\section{A. Intrinsic acoustic phonons}

For intrinsic $\mathrm{MoS}_{2}$ acoustic (AP) and optical (OP) phonons, the carrier-phonon coupling matrix element is given by [39]

$$
\left|\mathrm{g}_{\mathbf{q}, v}^{\lambda}\right|^{2}=\frac{\hbar}{2 \mathcal{A} \rho \omega_{\mathbf{q}, v}^{\lambda}}\left|M_{\mathbf{q}, \lambda}\right|^{2},
$$

where $\rho$ is the mass density and $M_{\mathbf{q}, \lambda}$ is the matrix element depending on the phonon mode index $\lambda(\lambda=\mathrm{LA}, \mathrm{TA}, \mathrm{LO}$, and TO) and the coupling mechanisms.

We consider acoustic phonons ( $v=\mathrm{AP}$ ) with linear dispersion $\omega_{\mathbf{q}, \lambda}=v_{s \lambda} q$, where $v_{s \lambda}$ is the velocity of the $\lambda$ th mode acoustic phonon with $\lambda=$ LA and TA. Due to the lack of inversion symmetry in the hexagonal lattice of monolayer $\mathrm{MoS}_{2}$, the coupling to the in-plane LA and TA phonons has contributions from both deformation potential (DP) and piezoelectric (PE) coupling mechanisms [37]. The matrix element for the DP coupling is given by

$$
M_{\mathbf{q}, \lambda}^{\mathrm{DP}}=\Xi_{\lambda} q,
$$

where $\Xi_{\lambda}$ is the effective deformation potential coupling constant. For the PE interaction, the matrix element is given by [37]

$$
M_{\mathbf{q}, \lambda}^{\mathrm{PE}}=\frac{e e_{11}}{\varepsilon_{0}} q \operatorname{erfc}\left(\frac{q \sigma}{2}\right)\left|A_{\lambda}(\mathbf{q})\right|,
$$

where $e_{11}$ is the piezoelectric constant, $\operatorname{erfc}(x)$ is the complementary error function, $\sigma$ is the effective width of the electronic wave functions, and $A_{\lambda}(\mathbf{q})$ is an anisotropy factor. In the long-wavelength limit, where $\operatorname{erfc}(q \sigma / 2) \sim 1[38,39]$ and $A_{\lambda}(\mathbf{q})=1 / \sqrt{2}$ [37], the matrix element for the piezoelectric interaction goes as

$$
M_{\mathbf{q}, \lambda}^{\mathrm{PE}} \approx \frac{e e_{11}}{\varepsilon_{0} \sqrt{2}} q
$$

Hence, the matrix element for acoustic phonon interaction in Eqs. (21) and (23) can be reduced as

$$
M_{\mathbf{q}, \lambda}^{\mu}=D_{\lambda}^{\mu} q, \quad \mu=\mathrm{DP}, \mathrm{PE},
$$

where $D_{\lambda}^{\mu}=\Xi_{\lambda}$ for $\mu=\mathrm{DP}$ and $D_{\lambda}^{\mu}=e e_{11} /\left(\varepsilon_{0} \sqrt{2}\right)$ for $\mu=$ PE. Thus, in the long-wavelength limit, the deformation potential and piezoelectric interactions of a 2D material depend on $q$ in the same way.

For quasielastic scattering on an acoustic phonon, since the energy of the acoustic phonon is much smaller than the separation energy, i.e., $\hbar \omega_{\mathbf{q}, \lambda} \ll E_{\alpha^{\prime}}-E_{\alpha}$, therefore $\hbar \omega_{\mathbf{q}, \lambda}$ can be neglected in the delta functions in Eq. (17). In this case, the approximation $N_{\mathbf{q}, \lambda}+1 \approx N_{\mathbf{q}, \lambda}=k_{\mathrm{B}} T / \hbar \omega_{\mathbf{q}, \lambda}$ could be made for the acoustic phonon distribution. Inserting Eq. (24) into Eq. (20) then into Eq. (17), the MOAC for quasielastic scattering on acoustic phonons is found to be

$$
\begin{aligned}
K_{\lambda}^{\mu}(\Omega)= & \mathcal{D}\left(\omega_{c}, \Omega\right) \frac{4 k_{\mathrm{B}} T\left(D_{\lambda}^{\mu}\right)^{2}}{\mathcal{A} \rho v_{s \lambda}^{2} a_{c}^{4}} \sum_{\alpha, \alpha^{\prime}}\left|\mathcal{B}_{\alpha \alpha^{\prime}}\right|^{2} f_{\alpha}\left(1-f_{\alpha^{\prime}}\right) \\
& \times \frac{\delta_{s s^{\prime}}}{L_{n, s}^{\eta, \tau} L_{n^{\prime}, s^{\prime}}^{\eta^{\prime}, \tau}}\left[\mathcal{F}_{\alpha, \alpha^{\prime}}^{(1)} \delta\left(X_{1}\right)+\mathcal{F}_{\alpha, \alpha^{\prime}}^{(2)} \delta\left(X_{2}\right)\right] .
\end{aligned}
$$

Here

$$
\begin{aligned}
\mathcal{F}_{\alpha, \alpha^{\prime}}^{(1)} & =2 m+j-\Lambda_{n, s}^{\eta, \tau} \Lambda_{n^{\prime}, s^{\prime}}^{\eta^{\prime}, \tau} \sqrt{m(m+j)}, \\
\mathcal{F}_{\alpha, \alpha^{\prime}}^{(2)}= & \frac{\alpha_{0}^{2}}{8 a_{c}^{2}}\left[2+6 m^{2}+j(j+6 m)\right. \\
& \left.-2 \Lambda_{n, s}^{\eta, \tau} \Lambda_{n^{\prime}, s^{\prime}}^{\eta^{\prime}, \tau}(2 m+j) \sqrt{m(m+j)}\right],
\end{aligned}
$$

and the argument of the delta functions

$$
X_{\ell}=E_{\alpha^{\prime}}-E_{\alpha}-\ell \hbar \Omega \text {. }
$$

Following the LL-broadening model [68,69], the delta functions in Eq. (25) are replaced by the Lorentzians of the widths $\gamma_{\alpha, \alpha^{\prime}}^{\mu, \lambda}$, namely $\delta(x)=\left(\gamma_{\alpha, \alpha^{\prime}}^{\mu, \lambda} / \pi\right) /\left[x^{2}+\left(\gamma_{\alpha, \alpha^{\prime}}^{\mu, \lambda}\right)^{2}\right]$, with

$$
\left(\gamma_{\alpha, \alpha^{\prime}}^{\mu, \lambda}\right)^{2}=\frac{k_{\mathrm{B}} T\left(D_{\lambda}^{\mu}\right)^{2}}{4 \pi \rho v_{s \lambda}^{2} a_{c}^{2}} \frac{\delta_{s s^{\prime}}}{L_{n, s}^{\eta, \tau} L_{n^{\prime}, s^{\prime}}^{\eta^{\prime}, \tau}} .
$$


In contrast to the previous work [8], where the spectral broadening is independent the band indices, here the Lorentzian widths vary with the initial and final states.

\section{B. Intrinsic optical phonons}

For optical phonon scattering $(v=\mathrm{OP})$, both the zero- and first-order deformation potential interactions are considered. Their respective matrix elements are given by [38]

$$
M_{\mathrm{q}, \lambda}=D_{\lambda}^{0} \text { and } M_{\mathrm{q}, \lambda}=D_{\lambda}^{1} q .
$$

Meanwhile, the coupling matrix element with 2D carriers for the polar LO phonon can be given by the Fröhlich interaction [7]

$$
g_{\mathrm{LO}}(q)=g_{\mathrm{Fr}} \operatorname{erfc}(q \sigma / 2),
$$

where $g_{\mathrm{Fr}}$ is the coupling constant and $\sigma$ is the effective width of the electronic Bloch states.

By means of the dispersionless optical phonons $\omega_{\mathbf{q}, \lambda}=$ $\omega_{\mathrm{HP}}$, the expression of the MOAC for inelastic scattering on a homopolar optical phonon is given as follows for the zeroorder deformation potential interaction:

$$
\begin{aligned}
K_{\mathrm{HP}}^{0}(\Omega)= & \mathcal{D}\left(\omega_{c}, \Omega\right) \frac{2 \hbar\left(D_{\mathrm{HP}}^{0}\right)^{2}}{\mathcal{A} \rho \omega_{\mathrm{HP}} a_{c}^{4}} \sum_{\alpha, \alpha^{\prime}}\left|\mathcal{B}_{\alpha \alpha^{\prime}}\right|^{2} f_{\alpha}\left(1-f_{\alpha^{\prime}}\right) \\
& \times \frac{\delta_{s s^{\prime}}}{L_{n, s}^{\eta, \tau} L_{n^{\prime}, s^{\prime}}^{\eta^{\prime} \tau}} N_{\mathrm{HP}}\left\{\mathcal{F}_{\alpha, \alpha^{\prime}}^{(1)}\left[\delta\left(Y_{1}^{-}\right)+e^{\hbar \omega_{\mathrm{HP}} / k_{B} T} \delta\left(Y_{1}^{+}\right)\right]\right. \\
& \left.+\mathcal{F}_{\alpha, \alpha^{\prime}}^{(2)}\left[\delta\left(Y_{2}^{-}\right)+e^{\hbar \omega_{\mathrm{HP}} / k_{B} T} \delta\left(Y_{2}^{+}\right)\right]\right\},
\end{aligned}
$$

where $N_{\mathrm{HP}}$ is is the equilibrium distribution function for the phonons $\omega_{\mathrm{HP}}$ given by the Bose-Einstein distribution function, $\mathcal{F}_{\alpha, \alpha^{\prime}}^{(1)}$ and $\mathcal{F}_{\alpha, \alpha^{\prime}}^{(2)}$ are defined by Eqs. (26) and (27), and $Y_{\ell}^{ \pm}$is given the same form as $\mathcal{X}_{\ell}^{ \pm}$shown in Eq. (19) but with $\hbar \omega_{\mathbf{q}, v}$ replaced by $\hbar \omega_{\mathrm{HP}}$. The delta functions in Eq. (32) imply the energy conservation law, which shows the resonant behavior at the phonon-assisted cyclotron resonance (PACR) condition in $\mathrm{MoS}_{2}$ based on the following selection rule:

$$
\ell \hbar \Omega=E_{\alpha^{\prime}}-E_{\alpha} \pm \hbar \omega_{\mathrm{HP}} .
$$

The corresponding Lorentzian widths for HP optical phonon scattering are given as follows:

$$
\left(\gamma_{\alpha, \alpha^{\prime}}^{ \pm, \mathrm{HP}}\right)^{2}=\frac{\hbar\left(D_{\mathrm{HP}}^{0}\right)^{2}}{4 \pi \rho \omega_{\mathrm{HP}} a_{c}^{2}} \frac{\delta_{s s^{\prime}}}{L_{n, s}^{\eta, \tau} L_{n^{\prime}, s^{\prime}}^{\eta^{\prime}, \tau}} N_{\mathrm{HP}}^{ \pm},
$$

which have the same form as those for acoustic phonon scattering. The result for the first-order deformation potential interaction can also be obtained in the same way (see the Appendix). In the case of the polar LO phonon interaction, the MOAC could not be computed analytically for the angular part of the $\mathbf{q}$ and it must be calculated numerically.

\section{Surface optical phonons}

For monolayer $\mathrm{MoS}_{2}$ on substrates (Table I), just like graphene $[15,44,71,72]$, the surface optical phonons $(v=$ SO) of the substrates interact with the carriers in $\mathrm{MoS}_{2}$ via an effective electric field $[67,73,74]$. Under the reasonable assumption of a dispersionless surface optical phonon
TABLE I. Material parameters for monolayer $\mathrm{MoS}_{2}$ used for numerical calculation.

\begin{tabular}{lcl}
\hline \hline Parameter & Symbol & \multicolumn{1}{c}{ Value } \\
\hline Lattice constant [9] & $a$ & $3.193 \AA$ \\
Effective electron mass [7] & $m^{*}$ & $0.84 \mathrm{~m}_{e}$ \\
Hopping integral [9] & $t$ & $1.1 \mathrm{eV}$ \\
Energy gap [9] & $\Delta$ & $1.66 \mathrm{eV}$ \\
Spin splitting energy [9,70] & $\lambda$ & $75 \mathrm{meV}$ \\
Mass density [7] & $\rho$ & $3.1 \times 10^{-7} \mathrm{~g} / \mathrm{cm}^{2}$ \\
Transverse sound velocity [7] & $c_{\mathrm{TA}}$ & $4.2 \times 10^{3} \mathrm{~m} / \mathrm{s}$ \\
Longitudinal sound velocity [7] & $c_{\mathrm{LA}}$ & $6.7 \times 10^{3} \mathrm{~m} / \mathrm{s}$ \\
Acoustic DP [7] & & \\
TA & $\Xi_{\mathrm{TA}}$ & $1.6 \mathrm{eV}$ \\
LA & $\Xi_{\mathrm{LA}}$ & $2.8 \mathrm{eV}$ \\
Piezoelectric constant [37] & $e_{11}$ & $3.0 \times 10^{-11} \mathrm{C} / \mathrm{m}$ \\
Optical DP [7] & & \\
Homopolar & $D_{\mathrm{HP}}^{0}$ & $4.1 \times 10^{8} \mathrm{eV} / \mathrm{cm}$ \\
TO & $D_{\mathrm{TO}}^{1}$ & $4.0 \mathrm{eV}$ \\
Fröhlich interaction (LO) [7] & & \\
Coupling constant & $g_{\mathrm{Fr}}$ & $98 \mathrm{meV}$ \\
Effective layer thickness & $\sigma$ & $4.41 \AA$ \\
Optical phonon energies [41] & & \\
TO & $\hbar \omega_{\mathrm{TO}}$ & $48.6 \mathrm{meV}$ \\
Homopolar & $\hbar \omega_{\mathrm{HP}}$ & $50.9 \mathrm{meV}$ \\
Polar LO & $\hbar \omega_{\mathrm{LO}}$ & $48.0 \mathrm{meV}$ \\
\hline \hline & & \\
\hline
\end{tabular}

$\omega_{\mathbf{q}, v}=\omega_{\mathrm{SO}}^{\lambda}$, with $\lambda=1,2$ presenting the first and second modes of the SO phonon [15], the MOAC can be treated semianalytically. The coupling matrix element is given as follows:

$$
\left|\mathrm{g}_{\mathbf{q}, \mathrm{SO}}\right|^{2}=e^{2} \mathcal{F}_{\lambda}^{2} \frac{e^{-2 q d}}{q},
$$

where $d$ is the equilibrium distance between the $\mathrm{MoS}_{2}$ sheet from the substrate, and the electron-phonon coupling parameter is expressed as [75-77]

$$
\mathcal{F}_{\lambda}^{2}=\frac{\hbar \omega_{\text {SO }}^{\lambda}}{2 \mathcal{A} \varepsilon_{0}}\left(\frac{1}{\chi_{\infty}+1}-\frac{1}{\chi_{0}+1}\right),
$$

where $\chi_{\infty}$ and $\chi_{0}$ are the high- and low-frequency dielectric constants of the substrate, respectively. These parameters as well as the energies of the SO phonon for some of the common substrates are displayed in Table II. For SO phonon scattering,

TABLE II. SO phonon modes for the substrates h-BN (taken from Ref. [44]), $\mathrm{HfO}_{2}, \mathrm{SiO}_{2}$ (taken from Ref. [74]), $\mathrm{SiC}, \mathrm{AlN}$, and $\mathrm{ZrO}_{2}$ (taken from Ref. [15]).

\begin{tabular}{lcccccc}
\hline \hline & $\mathrm{h}-\mathrm{BN}$ & $\mathrm{HfO}_{2}$ & $\mathrm{SiO}_{2}$ & $\mathrm{SiC}$ & $\mathrm{AlN}$ & $\mathrm{ZrO}_{2}$ \\
\hline$\chi_{0}$ & 5.09 & 22.0 & 3.90 & 9.7 & 9.14 & 24.0 \\
$\chi_{\infty}$ & 4.10 & 5.03 & 2.40 & 6.5 & 4.8 & 4.0 \\
$\hbar \omega_{\text {SO }}^{1}(\mathrm{meV})$ & 101.7 & 21.6 & 58.9 & 116.00 & 83.60 & 25.02 \\
$\hbar \omega_{\text {SO }}^{2}(\mathrm{meV})$ & 195.7 & 54.2 & 156.4 & 167.58 & 104.96 & 70.80 \\
\hline \hline
\end{tabular}



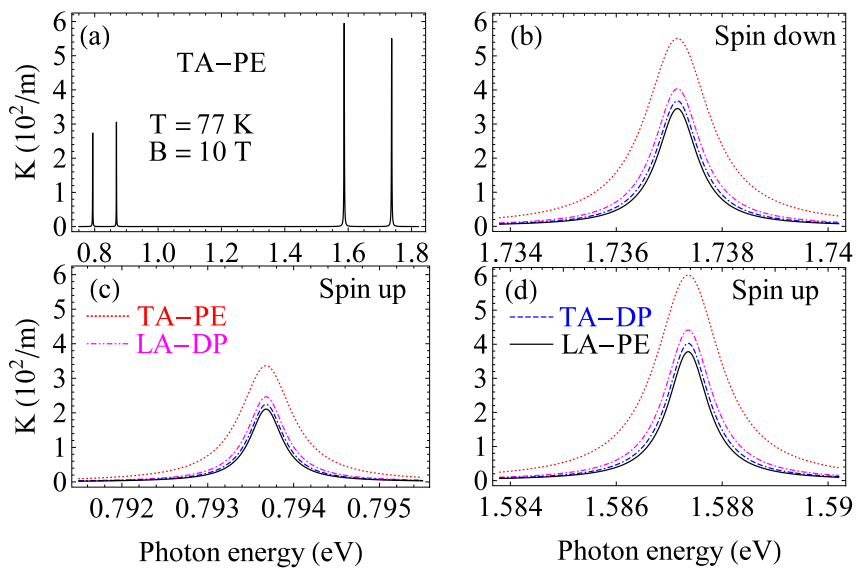

FIG. 2. Photon energy dependence of MOAC in monolayer $\mathrm{MoS}_{2}$ due to electron-acoustic phonons interaction: (a) individual piezoelectric scattering of the TA phonon; and different types of acoustic phonons: (b) one-photon, spin down, (c) two-photon, spin up, and (d) two-photon, spin up. The results are calculated at $B=10$ $\mathrm{T}$ and $T=77 \mathrm{~K}$.

the expression for the MOAC goes as

$$
\begin{aligned}
K_{\mathrm{SO}}^{\lambda}(\Omega)= & \mathcal{D}\left(\omega_{c}, \Omega\right) e^{2} \mathcal{F}_{\lambda}^{2} \frac{\sqrt{2}}{a_{c}^{3}} \sum_{\alpha, \alpha^{\prime}}\left|\mathcal{B}_{\alpha \alpha^{\prime}}\right|^{2} f_{\alpha}\left(1-f_{\alpha^{\prime}}\right) \\
& \times N_{\mathrm{SO}}^{\lambda}\left\{\mathcal{G}_{\alpha, \alpha^{\prime}}^{(1)}\left[\delta\left(Z_{1}^{-}\right)+e^{\hbar \omega_{\mathrm{SO}}^{\lambda} / k_{B} T} \delta\left(Z_{1}^{+}\right)\right]\right. \\
& \left.+\frac{\alpha_{0}^{2}}{8 a_{c}^{2}} \mathcal{G}_{\alpha, \alpha^{\prime}}^{(2)}\left[\delta\left(Z_{2}^{-}\right)+e^{\hbar \omega_{\mathrm{SO}}^{\lambda} / k_{B} T} \delta\left(Z_{2}^{+}\right)\right]\right\}
\end{aligned}
$$

Here we introduced the following dimensionless integral with $p=1$ and 2 :

$$
\mathcal{G}_{\alpha, \alpha^{\prime}}^{(p)}=\int_{0}^{\infty} u^{p-1 / 2} e^{-2 d \sqrt{2 u} / a_{c}}\left|J_{\alpha, \alpha^{\prime}}(u)\right|^{2} d u,
$$

which can be evaluated numerically, and $Z_{\ell}^{ \pm}$is given the same form as $\mathcal{X}_{\ell}^{ \pm}$shown in Eq. (19) but with $\hbar \omega_{\mathbf{q}, v}$ replaced by $\hbar \omega_{\mathrm{SO}}^{\lambda}$. The corresponding Lorentzian widths are given as follows:

$$
\left(\gamma_{\alpha, \alpha^{\prime}}^{ \pm, \mathrm{SO}, \lambda}\right)^{2}=\frac{\mathcal{A} e^{2} \mathcal{F}_{\lambda}^{2} \mathcal{G}_{\alpha, \alpha^{\prime}}^{(0)}}{4 \pi \sqrt{2} a_{c}} N_{\mathrm{SO}}^{ \pm, \lambda},
$$

where $\mathcal{G}_{\alpha, \alpha^{\prime}}^{(0)}$ is defined in Eq. (38) with $p=0$.

\section{NUMERICAL RESULTS AND DISCUSSION}

For the numerical calculation, we put the carrier density $n_{e}=10^{13} \mathrm{~cm}^{-2}$ [78] leading to the Fermi energy $E_{F}=$ $25 \mathrm{meV}$ [37], which is located between the conduction and valence bands. This means that the main transition arises from $n=0$ to $n^{\prime}=1$ states. The other parameters for intrinsic acoustic and optical phonons are shown in Table I and $\alpha_{0}=5 \mathrm{~nm}$ [45]. Since the $K$ and $K^{\prime}$ valleys are symmetric (see Fig. 1), we only consider $\tau=\tau^{\prime}=+1$, and in the following the indices $\tau$ and $\tau^{\prime}$ will be ignored.

\section{A. Intrinsic acoustic phonons}

Figure 2(a) shows the MOAC in monolayer $\mathrm{MoS}_{2}$ as a function of photon energy due to acoustic phonon scattering by the PE coupling of a TA phonon. We can clearly see four pronounced absorption peaks, which correspond to interband transitions. Unlike in graphene [59] and other graphenelike 2D systems $[48,52,66]$, the large band gap and strong SOC in the $\mathrm{MoS}_{2}$ spectrum have main implications for the absorption peaks seen in the MOAC. The large band gap is the cause of the fact that the interband transition peaks occur in the visible frequency range, agreeing with the previous work [12]. Meanwhile, the strong SOC results in the splitting of the LLs, which is the cause of the separated energies of the spin up and down states. In specific terms, the first and second peaks are for the two-photon absorption process $(\ell=2)$, with spin up and down, respectively, while the third and fourth peaks correspond to the one-photon process $(\ell=1)$ with spin up and down states, respectively. The peak values in the case of two-photon absorption are about $50 \%$ of those in the case of one photon. This implies that the two-photon process has a significant contribution to the total and cannot be neglected when investigating the optical transport properties of monolayer $\mathrm{MoS}_{2}$. We also note that the MOAC due to both one and two-photon absorption processes for the spin up state is always higher than those for the spin down one.

To investigate the interaction between electrons and different kinds of acoustic phonons, in Figs. 2(b), 2(c), and 2(d) we show the MOAC as a function of photon energy for both DP and PE coupling as well as for both spin up [Figs. 2(c) and 2(d)] and down [Fig. 2(b)] states at $B=10 \mathrm{~T}$ and $T=77 \mathrm{~K}$. Although we only focus on the one-photon absorption process [see Figs. 2(b) and 2(d)], the results could be also validated for the two-photon case [see Fig. 2(c)]. Indeed, in Fig. 2(d), the main resonant peaks are located at $\hbar \Omega=1.5874 \mathrm{eV}$, and satisfy the resonant condition in Eq. (28) with $(n=0, s=$ $+1, \eta=-1),\left(n^{\prime}=1, s^{\prime}=+1, \eta^{\prime}=+1\right)$, and $\ell=1$. In this work, we neglect the effect of screening [37,39], which has been demonstrated to reduce the absorption coefficient [39]. This means that for both the DP and PE coupling, phonon interactions are taken to be unscreened. Unlike free-carrier optical absorption [39], here we found that the contribution due to the TA phonon via PE coupling dominates the others. This disagreement might come from the fact that the effect of screening is not considered. In specific terms of PE coupling, the contribution of TA phonons is about 1.6 times larger than that of LA phonons, which can result from smaller TA phonon velocity when the MOAC is nearly proportional to $v_{s \lambda}^{-2}$ [see Eq. (25)], agreeing with the previous work [39]. It is also found that in DP coupling, the LA mode provides a larger contribution in comparison with the TA mode consisting of its larger coupling strength. This result is in good agreement with that for the scattering rate [41], whereas it is in contrast to the cooling power, where the TA mode is dominant [38]. A similar result but with slightly smaller amplitudes of the resonant peaks is observed with the spin down state as shown in Fig. 2(b). It can be seen that the difference between the absorption by spin up and spin down electrons is small, and their quality is similar. Therefore, in the following, we would only focus on the spin up case, and the results could be validated for the spin down case. 

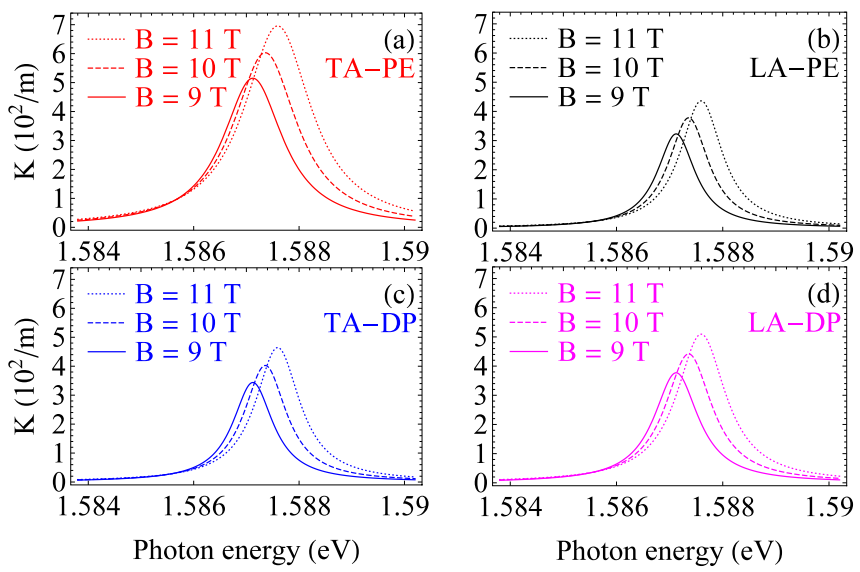

FIG. 3. MOAC in monolayer $\mathrm{MoS}_{2}$ is showed as a function of photon energy for different values of magnetic field: (a) TA-PE, (b) LA-PE, (c) TA-DP, and (d) LA-DP phonons. The results are calculated for the spin up case at $T=77 \mathrm{~K}$.

Figure 3(a) shows the photon energy-dependent MOAC for monolayer $\mathrm{MoS}_{2}$ due to a TA phonon via PE coupling at three different values of the magnetic field. For each value of $B=9,10$, and $11 \mathrm{~T}$, the spectrum exhibits a single absorption peak, with a maximum at a photon energy of $1.5871,1.5874$, and $1.5876 \mathrm{eV}$ and a width of $1.3931,1.4684$, and $1.5401 \mathrm{meV}$ (FWHM), respectively. The main trend that can be observed here is that with increasing magnetic field the resonant peaks shift to higher energies and their amplitudes increase. This behavior of the MOAC is similar to that in a semiconductor quantum well [79] and in graphene [44,45,62]. This can be qualitatively explained in the one-particle picture as originating from the LL spectrum as follows: With the increase of the magnetic field, the LL spacing increases giving upswing to a higher energy of the absorbed photon. Besides, the increase of the amplitudes of the interband transition peaks with increasing magnetic field can be interpreted as reducing the magnetic length $a_{c}$. We can see from Eq. (25) that the MOAC is nearly proportional to $a_{c}^{-4} \sim B^{2}$. Consequently, with increasing magnetic field the magnetic length reduces giving rise to an increase of the amplitudes of the resonant peaks. Similar behavior of the MOAC is observed for the case of LA-PE, TA-DP, and LA-DP phonons shown in Figs. 3(b)-3(d), respectively, but with smaller amplitudes. While the major trends in Fig. 3 can be clearly understood from the one-particle picture, the electron-phonon scattering still plays an intense role and is needed to explain the broadening of the resonant peaks with magnetic field.

In order to gain insight into the nature of electron-phonon scattering in monolayer $\mathrm{MoS}_{2}$ in the presence of a magnetic field, we measured the FWHM as a function of the magnetic field. Figure 4 shows the dependence of the FWHM on the magnetic field at $T=77 \mathrm{~K}$ for both DP and PE coupling in the case of spin up. The result is calculated for both the one- and two-photon absorption processes. With increasing magnetic field, the FWHM of the resonant peaks increases in both one- and two-photon absorption processes as well as for both DP and PE coupling. The $\sqrt{B}$-dependent FWHM can result from the broadening of the LLs [80]. This is

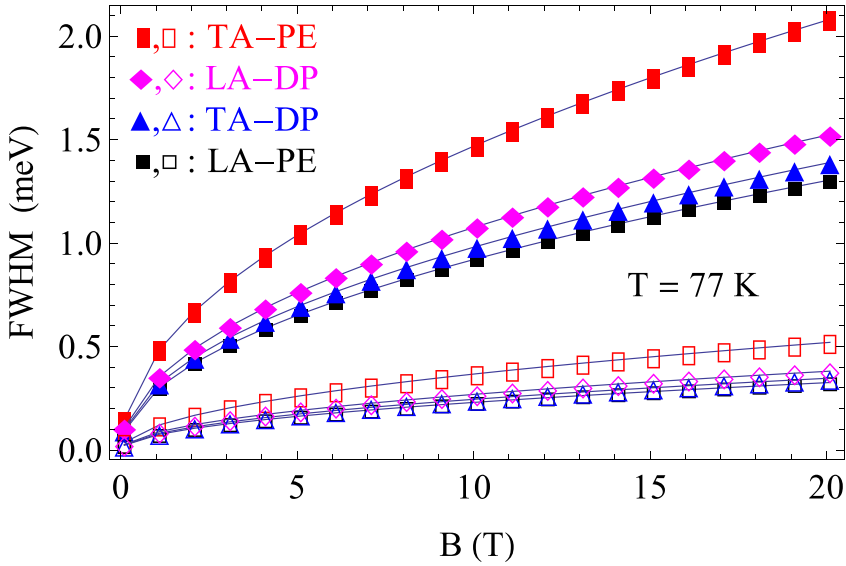

FIG. 4. Magnetic field dependence of FWHM of main peaks in monolayer $\mathrm{MoS}_{2}$ due to electron-acoustic phonon scattering for the spin up case at $T=77 \mathrm{~K}$. The full and empty dots correspond to the one- and two-photon absorption processes.

in overall good agreement with the result observed in the conventional 2D electron gas [81], in 2D graphite systems [82], and in graphene [83]. It is noted that the FWHM has a close relationship with Lorentzians width, proportional to the ratio $D_{\lambda}^{\mu} / v_{s \lambda}$ [see Eq. (29)]. Since the piezoelectric constant $e_{11}=3.0 \times 10^{-11} \mathrm{C} / \mathrm{m}$ [37] leads to a potential $D_{\lambda}^{\mathrm{PE}}=2.4 \mathrm{eV}$, a TA phonon via PE coupling with the strongest ratio $D_{\lambda}^{\mu} / v_{s \lambda}$ shows the largest FWHM among the four, while an LA-PE phonon shows the smallest. This is attributed to the fact that in the first case, the probability of electron-phonon scattering is the strongest. For example, at $B=10 \mathrm{~T}$, the FWHM via a one-photon absorption process due to a TA phonon via PE coupling is about $1.4684 \mathrm{meV}$, whereas they are 1.0753 , 0.9802 , and $0.9205 \mathrm{meV}$ for LA-DP, TA-DP, and LA-PE phonons, respectively. Moreover, in each phonon mode the distribution of the two-photon absorption process is always smaller than that of the one-photon case. For instance, at $B=10 \mathrm{~T}$, the distribution of the two-photon process is about $25 \%$ of that of the one-photon case in all four modes of acoustic phonons. This means the two-photon absorption process is strong enough to be detected and should be considered in investigating the MOAC in monolayer $\mathrm{MoS}_{2}$.

Figure 5 shows the dependence of the individual MOAC in monolayer $\mathrm{MoS}_{2}$ on photon energy at different values of temperature for both DP and PE coupling at $B=10 \mathrm{~T}$. The main peaks occur at $\hbar \Omega=1.5874 \mathrm{eV}$ as mentioned in Figs. 2 and 3. The nearby peaks at $\hbar \Omega=1.5921 \mathrm{eV}$ result from the $n=1 \rightarrow n^{\prime}=2$ transition, which are much smaller in comparison to the main peaks. With increasing temperature, the resonant peaks give a rising spectral amplitude and become broader while their positions are maintained. The rise of spectral magnitude is similar to the scattering rate [37], cooling power [38], free-carrier optical absorption [39], and phononassisted cyclotron resonance [84]. For a TA-PE phonon [see Fig. 5(a)], in particular, the strong rise in the peak value of the MOAC from $1.37 \times 10^{2} / \mathrm{m}$ at $T=4 \mathrm{~K}$ to $1.19 \times 10^{3} / \mathrm{m}$ at $T=300 \mathrm{~K}$ is a result of increased electron-phonon scattering at higher temperature. The positions of resonant peaks are governed by the arguments of the delta functions in Eq. (28), in 

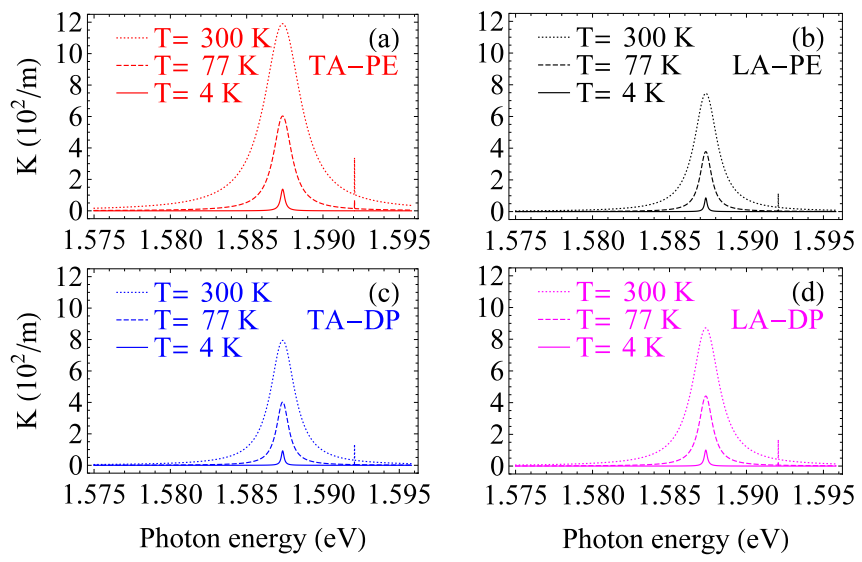

FIG. 5. MOAC in monolayer $\mathrm{MoS}_{2}$ is shown as a function of photon energy for different temperatures: (a) TA-PE, (b) LA-PE, (c) TA-DP, and (d) LA-DP phonons. The results are calculated for the spin up case at $B=10 \mathrm{~T}$.

which the absorbed photon energies must satisfy the condition $\ell \hbar \Omega=E_{\alpha^{\prime}}-E_{\alpha}$. Therefore, the temperature independence of the arguments of the delta functions leads to an unchanged position of the resonant peaks when the temperature increases. And the broadening of the resonant peaks originates from the increase of the probability of electron-phonon scattering at higher temperatures, which is reflected in the increase of the FWHM.

Figure 6 shows the FWHM of the main resonant peaks in Fig. 5 as a function of temperature for both DP and PE coupling at $B=10 \mathrm{~T}$. The FWHM in monolayer $\mathrm{MoS}_{2}$ can be governed by both the electron-phonon scattering and temperature. Besides strongly increasing with temperature, the FWHM is also dependent on the phonon modes and on the coupling mechanisms. The square root of $T$ dependence of the FWHM originates from the Lorentzians width in Eq. (29) which increases with $\sqrt{T}$. Meanwhile, the effect of phonon modes and coupling mechanisms on the FWHM is expressed

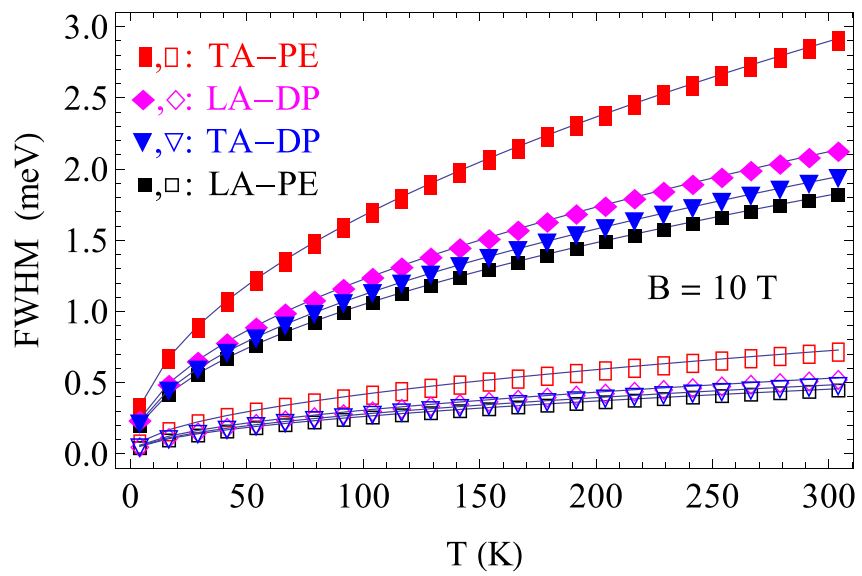

FIG. 6. Temperature dependence of FWHM of main peaks in monolayer $\mathrm{MoS}_{2}$ due to electron-acoustic phonon scattering for the spin up case at $B=10 \mathrm{~T}$. The full and empty dots correspond to the one- and two-photon absorption processes.
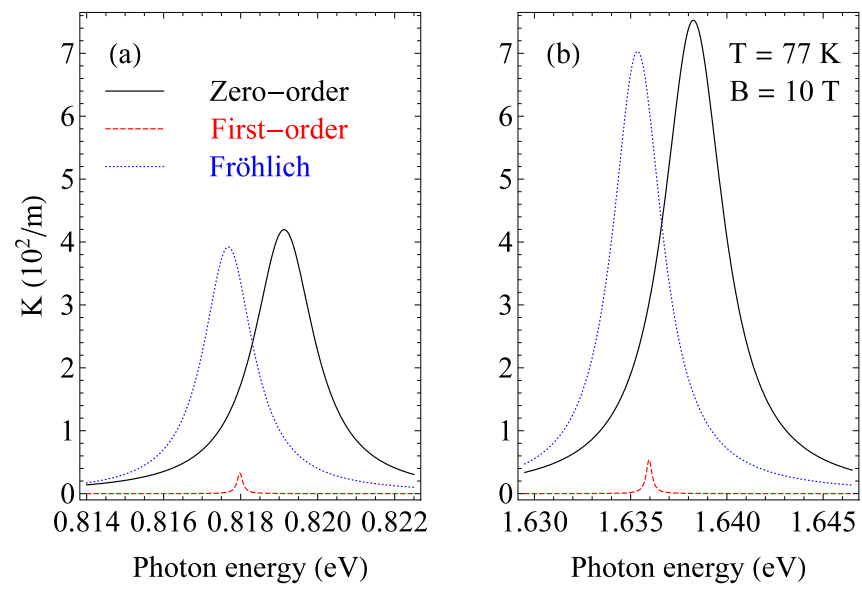

FIG. 7. MOAC for the different electron-optical phonon couplings as a function of photon energy at $T=77 \mathrm{~K}$ and $B=10$ T. The left (a) and right (b) panels correspond, respectively, to the two-photon and one-photon absorption processes and differ only in the photon energy range ( $x$ axis).

through the ratio $D_{\lambda}^{\mu} / v_{s \lambda}$. Similar to the result mentioned in Fig. 4, with the strongest ratio, the TA-PE phonon displays once again the strongest FWHM while the LA-PE phonon shows the smallest one.

\section{B. Intrinsic optical phonons}

The resulting MOAC for zero-order, first-order optical deformation potentials, and the Fröhlich interaction for the spin up case are shown in Fig. 7 as a function of the photon energy at $T=77 \mathrm{~K}$ and $B=10 \mathrm{~T}$. These peaks are due to PACR, which represents the resonance transfer of electrons with absorption of one [Fig. 7(b)] and/or two photons [Fig. 7(a)] accompanied by the absorption/emission of phonons. While the zero- and first-order optical deformation potential (ODP) couplings correspond to the interaction with the homopolar mode and the polar TO phonon, respectively, the Fröhlich interaction corresponds to the polar LO phonon. In general, the zero-order ODP and the Fröhlich interaction dominate the MOAC to the optical phonons, while the firstorder ODP is the weakest. This result is in good agreement with that calculated for scattering rate [7] and cooling power [38]. The high MOAC for the homopolar mode stems from its strong zero-order deformation potential coupling, which originates from its characteristic lattice vibration polarized in out-of-plane direction [7], where two S-atoms oscillate out of phase with each other and the Mo atom is in its steady state [41]. The position of the resonant peak depends on the phonon energies and is defined through Eqs. (33) and (A2). With the largest phonon energy (see Table I), the homopolar mode (zero-order) gives the largest shift towards higher energies, while the LO phonon (Fröhlich) gives the smallest shift among the three.

In Figs. 8 and 9 we show the contribution to the MOAC and FWHM from the individual coupling mechanisms at $T=77 \mathrm{~K}$. Via the interband transitions from occupied to unoccupied states through the absorption of one and two photons, the effect of a magnetic field on these features is 

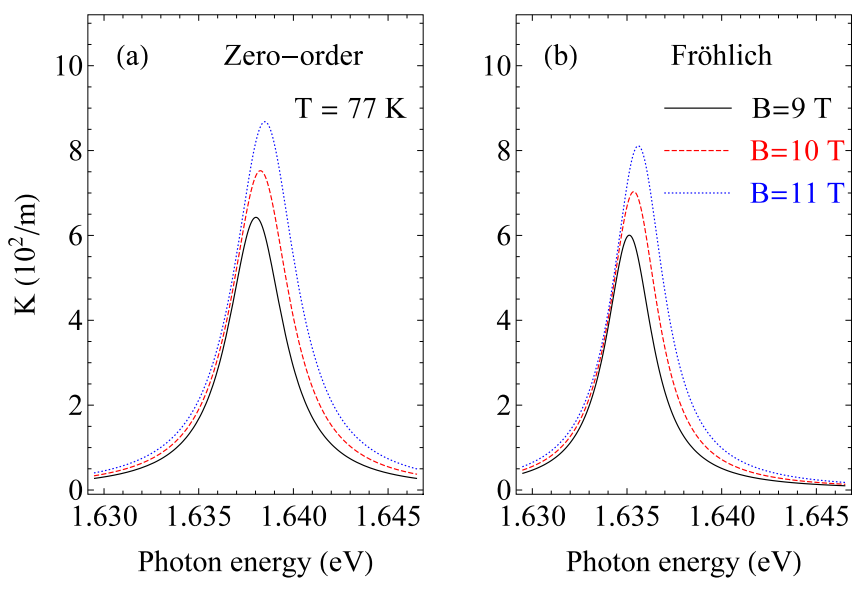

FIG. 8. MOAC for different electron-optical phonon couplings is showed as a function of photon energy for different magnetic fields: (a) Zero-order ODP and (b) Fröhlich. The results are calculated for the spin up case at $T=77 \mathrm{~K}$.

similar to those displayed in Figs. 3 and 4 due to acoustic phonon scattering but with a slightly higher magnitude. For the zero-order ODP and Fröhlich interaction, the $\sqrt{B}$-dependent FWHM is similar to that for acoustic phonons because of their identical form for the coupling matrix element. Whereas, in the case of first-order ODP, the linearly field dependent FWHM results from the fact that the Lorentzians width is proportional to $a_{c}^{-2} \sim B$ [see Eq. (A3)].

The effect of temperature on the MOAC and FWHM due to optical phonon scattering is illustrated in Figs. 10 and 11. We found that temperature does not impact the position but impacts the magnitude and the broadening of resonant peaks. As seen in Fig. 10, with increasing temperature, the resonant peaks become slightly broader and give a rise in spectral magnitude. The broadening of the resonant peak with the increase of temperature is reflected in the increase of the FWHM as displayed in Fig. 11. Unlike the case of acoustic phonon scattering (see Figs. 5 and 6), temperature weakly

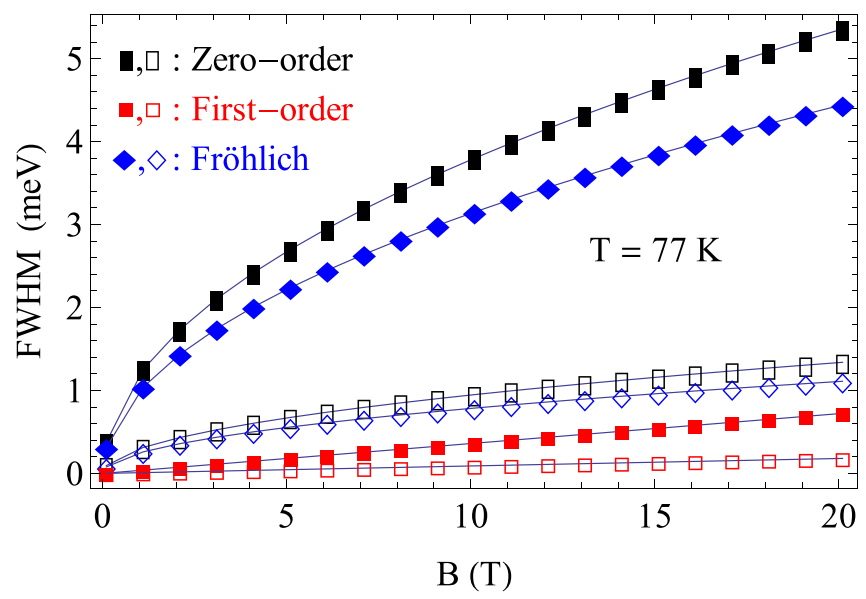

FIG. 9. Magnetic field dependence of FWHM of main peaks in monolayer $\mathrm{MoS}_{2}$ due to electron-optical phonon scattering for the spin up case at $T=77 \mathrm{~K}$. The full and empty dots correspond to the one- and two-photon absorption processes.
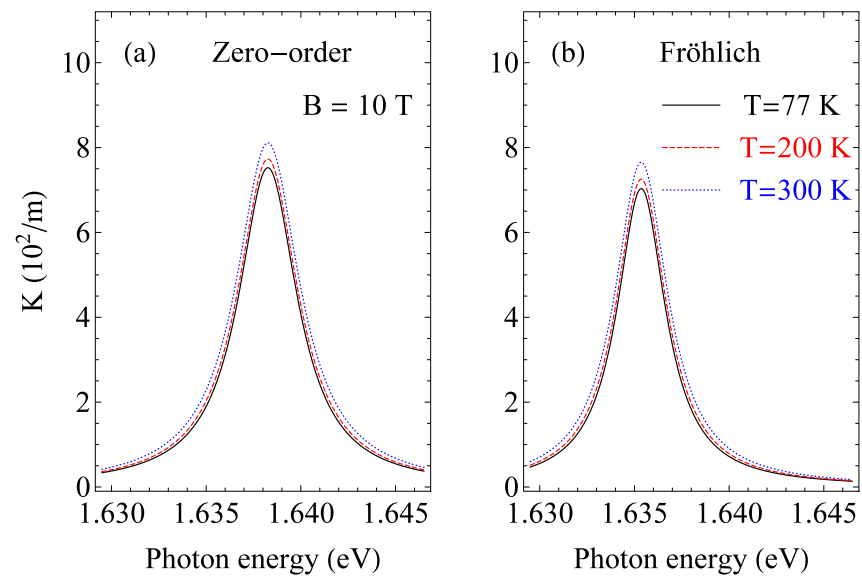

FIG. 10. MOAC for different electron-optical phonon couplings shown as a function of photon energy for different temperatures: (a) Zero-order ODP and (b) Fröhlich. The results are calculated for spin up at $B=10 \mathrm{~T}$.

affects FWHM through the slight dependence on temperature of the number of optical phonons. In the low-temperature regime, the effect of the optical phonons is not clear and the role of acoustic phonons is dominant. At higher temperatures, zero-order ODP and polar optical scattering via the Fröhlich interaction become dominant. Whereas, the first-order ODP only plays a minor role in the low field and the low-temperature regimes. This is in good agreement with previous results for the scattering rates [7].

\section{Surface optical phonons}

To illustrate the contribution to the MOAC of the two individual SO phonon modes as well as of the two-photon process, in Figs. 12(a) and 12(b) we show the MOAC as a function of photon energy for $\mathrm{MoS}_{2}$ on several substrates at $T=77 \mathrm{~K}, d=0.4 \mathrm{~nm}$, and $B=10 \mathrm{~T}$. It is found that

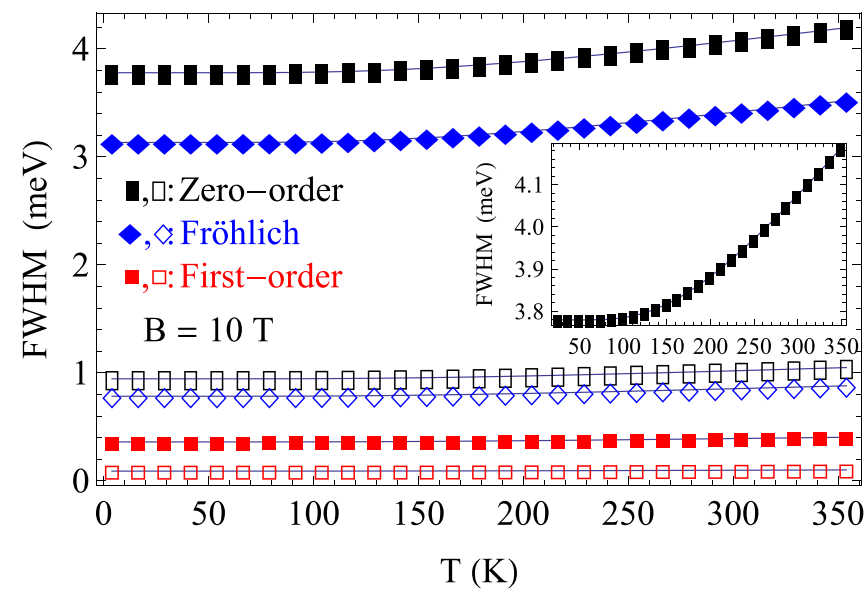

FIG. 11. Temperature dependence of FWHM of main peaks in monolayer $\mathrm{MoS}_{2}$ due to electron-optical phonon scattering for the spin up case. The full and empty dots correspond to the one- and two-photon absorption processes. The inset is zoomed in for the zero-order ODP. 

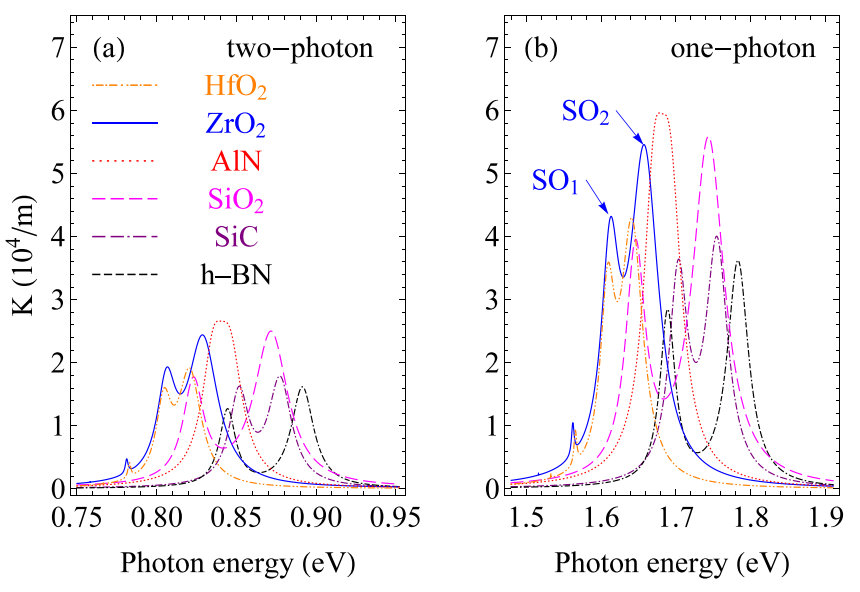

FIG. 12. Photon energy dependence of MOAC for $\mathrm{MoS}_{2}$ on several substrates due to electron-SO phonon interaction. The results are calculated for spin up at $T=77 \mathrm{~K}, d=0.4 \mathrm{~nm}$, and $B=10$ T. The left (a) and right (b) panels correspond, respectively, to the two-photon and one-photon absorption processes and differ only in the photon energy range.

despite their small energy, SO phonons play an important role in electron transport via enhancing SO phonon scattering if monolayer $\mathrm{MoS}_{2}$ is in close to high- $\kappa$ dielectrics [67]. The evidence is that the values of the MOAC due to electron-SO phonon scattering shown in Fig. 12 are much bigger than those due to the interaction between electron and the intrinsic acoustic (see Fig. 2) or optical phonons (see Fig. 7).

The resonant peaks appearing in each curve in Fig. 12 represent the PACR transfer of electrons from $n=0$ to $n^{\prime}=1$ states with absorption of photons accompanied by the absorption/emission of SO phonons. It is clear that, in comparison with the $\mathrm{SO}_{1}$ mode, an $\mathrm{SO}_{2}$ phonon with higher energy displays a stronger scattering with electrons, which is reflected in the MOAC peaks being higher, and also gives a shift of the peak position towards higher energies. An example is shown in Fig. 12(b) for $\mathrm{MoS}_{2}$ on a $\mathrm{ZrO}_{2}$ substrate (blue curve): the peaks at $\hbar \Omega=1.612$ and $1.658 \mathrm{eV}$ correspond to transitions due to $\mathrm{SO}_{1}$ and $\mathrm{SO}_{2}$ phonon scattering, respectively; and the magnitude of the peak due to $\mathrm{SO}_{1}$ phonon scattering is about $79 \%$ of that due to the $\mathrm{SO}_{2}$ phonon. In Fig. 12(a) we also obtained similar results for the two-photon absorption process, but with smaller values of the resonant peaks. These results are in good agreement with those calculated for graphene on polar substrates [45].

Another important feature is that the substrates strongly affect the MOAC spectra not only in magnitude but also in the position of resonant peaks. $\mathrm{SiO}_{2}$, with the highest electron-SO phonon coupling parameter $\left(\mathcal{F}_{\lambda}\right)$, shows the strongest electronSO phonon scattering, which is reflected in the highest MOAC magnitude, followed by that of the substrate $\mathrm{ZrO}_{2}$. Meanwhile, h-BN presents the lowest magnitude. Exceptionally, in the case of AlN, since the energies of the two SO phonon modes are nearly equal to each other, the positions of the resonant peaks due to these two SO phonon modes almost coincided. That is the reason why the magnitude is observed to be highest in this case although the electron-SO coupling is not the strongest. Besides, with the smallest SO phonon energies the position
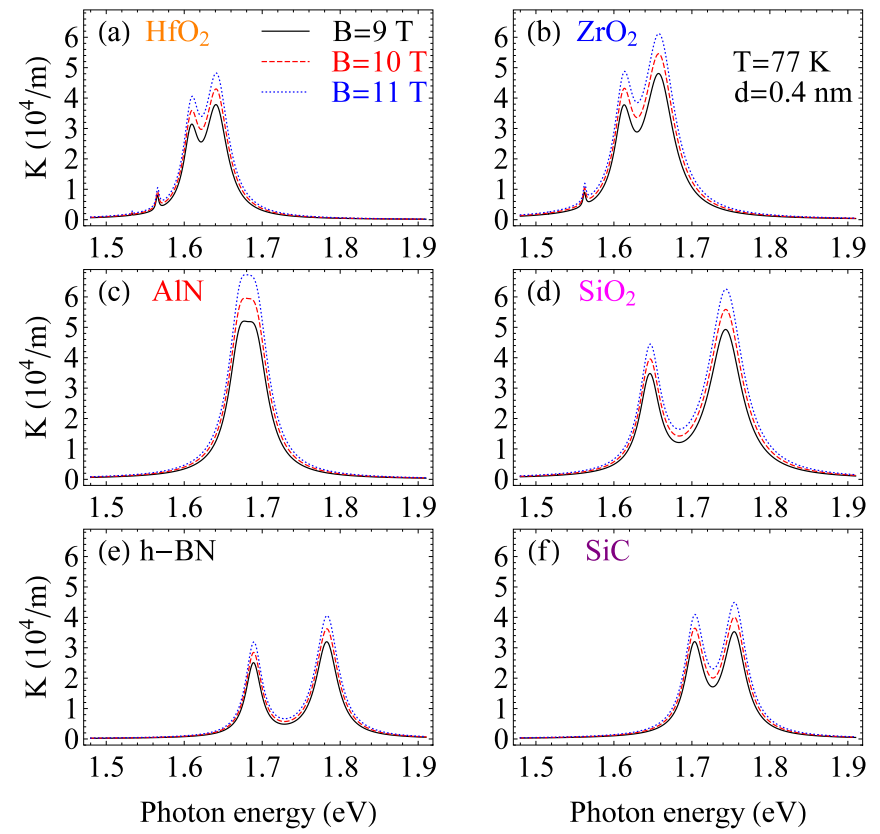

FIG. 13. Photon energy dependence of MOAC for $\mathrm{MoS}_{2}$ on several substrates due to electron-SO phonon interaction at different values of magnetic field $B$. The results are calculated for spin up at $T=77 \mathrm{~K}$ and $d=0.4 \mathrm{~nm}$.

of the resonant peaks in the case of $\mathrm{HfO}_{2}$ shifts to the lowest energy, while h-BN shows the largest among the six substrates.

To investigate the effect of a magnetic field on the absorption spectrum, in Figs. 13 and 14 we display the magnetic field dependence of MOAC and FWHM for $\mathrm{MoS}_{2}$ on many different substrates. The general trends observed in Fig. 13, for all six substrates, are that with increasing magnetic field the position of the resonant peaks move slightly toward higher energies (blueshift) and their amplitudes increase. These are in good agreement with those reported previously for the magneto-optical conductivity [44] and the phonon-assisted

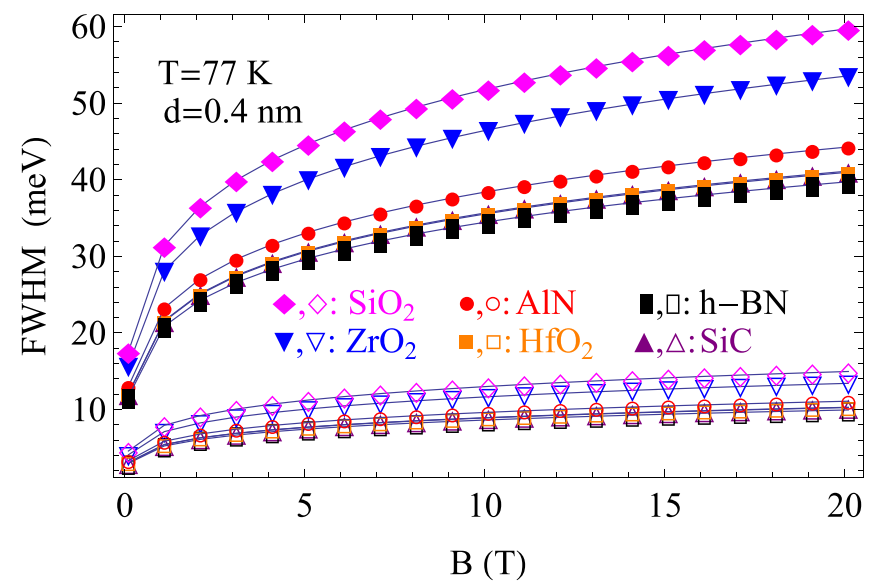

FIG. 14. Magnetic field dependence of FWHM for $\mathrm{MoS}_{2}$ on several substrates due to electron-SO phonon interaction. The results are calculated for spin up at $T=77 \mathrm{~K}$ and $d=0.4 \mathrm{~nm}$. The full and empty dots correspond to the one- and two-photon absorption processes, respectively. 

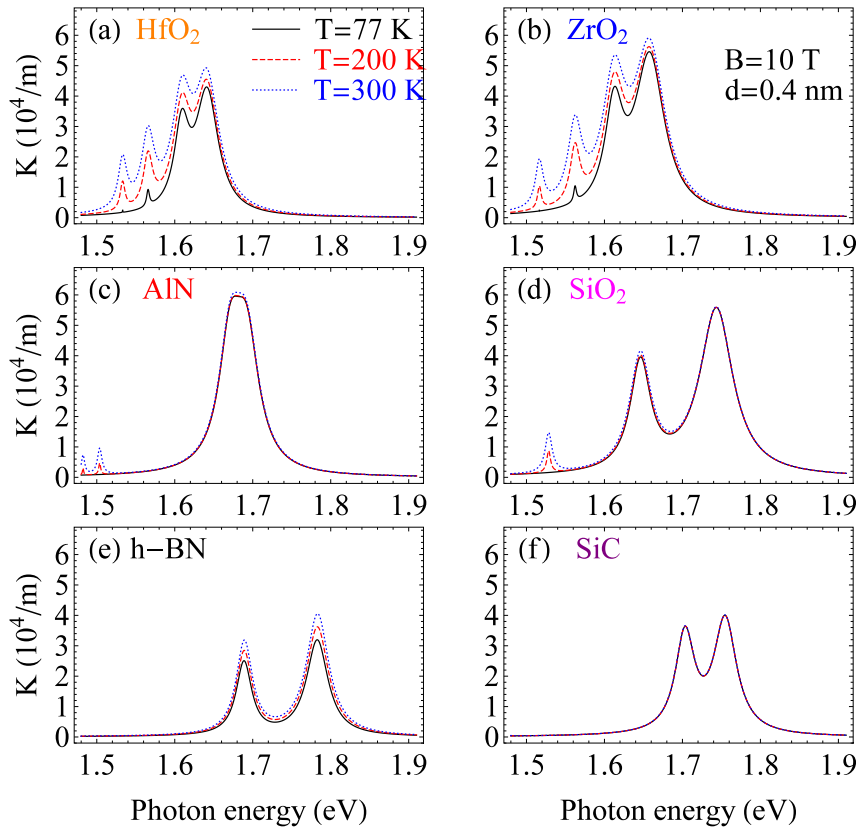

FIG. 15. Photon energy dependence of MOAC for $\mathrm{MoS}_{2}$ on several substrates due to electron-SO phonon interaction at different temperatures $T$. The results are calculated for spin up at $d=0.4 \mathrm{~nm}$ and $B=10 \mathrm{~T}$.

cyclotron resonance [45] in a graphene/polar substrate, as well as for the magneto-optical transitions [42] and magnetophonon resonance [8] in $\mathrm{MoS}_{2}$ /polar substrate heterostructures. These can be qualitatively explained by the fact that, as the magnetic field increases, (i) the Landau level spacing increases (see Fig. 1) which gives rise to a higher energy for the absorbed photons; and (ii) the decrease of magnetic length results in the enhancement of the amplitudes of resonant peaks.

The effect of different substrates on the absorption spectrum of $\mathrm{MoS}_{2}$ is also displayed in Fig. 13. It is found that the resonant feature of the MOAC in these substrates is the same. However, because of their larger SO phonon energies, the dielectrics $\mathrm{AlN}, \mathrm{SiO}_{2}, \mathrm{~h}-\mathrm{BN}$, and $\mathrm{SiC}$ play a minor role in comparison with $\mathrm{HfO}_{2}$ and $\mathrm{ZrO}_{2}$.

The dependence of the FWHM on the magnetic field for $\mathrm{MoS}_{2}$ on different substrates is shown in Fig. 14. The features of the FWHM are similar to these of other intrinsic acoustic and optical phonon modes. However, due to their strong electron-SO phonon couplings, the FWHM induced by $\mathrm{SO}$ phonons is bigger than that due to intrinsic acoustic and optical phonons. Once again, we can see that with the highest electron-SO phonon scattering, $\mathrm{SiO}_{2}$ shows the biggest FWHM, while h-BN displays the smallest one among the six substrates. Besides, the $\sqrt{B}$-dependent behavior of FWHM is similar to that in graphene $[45,83,85]$, which can result from the broadening of the Landau levels [80].

In Fig. 15 we now turn our attention to study the temperature dependent MOAC for $\mathrm{MoS}_{2}$ on different substrates. Comparing six substrates, one can discern that with the large low-frequency dielectric constant and small SO phonon energies, $\mathrm{HfO}_{2}$ and $\mathrm{ZrO}_{2}$ are strongly affected by temperature, especially for the absorbed SO phonon peaks [two leftmost

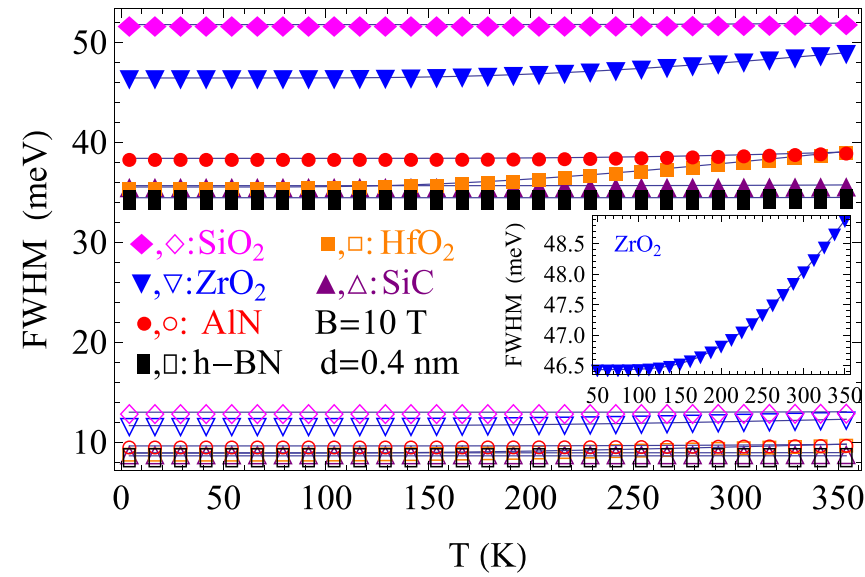

FIG. 16. Temperature dependence of FWHM for $\mathrm{MoS}_{2}$ on several substrates due to electron-SO phonon interaction. The results are calculated for spin up at $B=10 \mathrm{~T}$ and $d=0.4 \mathrm{~nm}$. The full and empty dots correspond to the one- and two-photon absorption processes, respectively. The inset is zoomed in for the case of the $\mathrm{ZrO}_{2}$ substrate.

peaks of each curve in Figs. 15(a) and 15(b)]. Besides, as can be seen in Fig. 15, which focuses on the optical transition from $n=0$ to $n^{\prime}=1$, the absorption peaks become broader and enhance spectral amplitude with the increasing temperature. This enhancement spectral weight is opposite to previously reported for graphene on polar substrates [44,45]. This difference comes from the position of the Fermi level: in the case of graphene its position is completely in the conduction band, while in $\mathrm{MoS}_{2}$ it lies between the conduction and valence bands. Therefore, in $\mathrm{MoS}_{2}$ there are no new thermally exited transitions as found in graphene [44]. Furthermore, the broadening of the absorption peaks comes from the increase of SO phonons and thus the probability of electron-SO phonon scattering with increasing temperature, which will be reflected in the enhancement of the FWHM as shown in Fig. 16.

Figure 16 shows the dependence of the FWHM on the temperature for $\mathrm{MoS}_{2}$ on several different substrates at $B=10$ $\mathrm{T}$ and $d=0.4 \mathrm{~nm}$. Similar to the case of intrinsic optical phonons, the slightly increasing FWHM comes from the enhancement of the number of SO phonons when temperature increases. It is clear that the role of the SO phonon is important only at high temperature, the unchanged behavior of the FWHM in the low-temperature range as shown in the inset of Fig. 16 is the evidence. Besides, due to the small SO phonon energies, the FWHM for $\mathrm{ZrO}_{2}$ and $\mathrm{HfO}_{2}$ substrates increase more quickly, in the high-temperature regime, than that for the other substrates. This result in close agreement with the previous experimental report made for graphene on a $\mathrm{SiC}$ substrate [85], and the theoretical result for graphene on polar substrates [45].

The importance of SO phonon scattering can also be observed in Fig. 17, which displays the dependence of the MOAC on photon energy for different values of the distance $d$. As can be seen in Fig. 17, the MOAC reduces with increasing distance $d$ due to the decreasing coupling strength between electrons and SO phonons [42] whence the decreasing SO phonon scattering rate, which has been demonstrated in previous studies $[15,86]$. These originate from the reduction of 

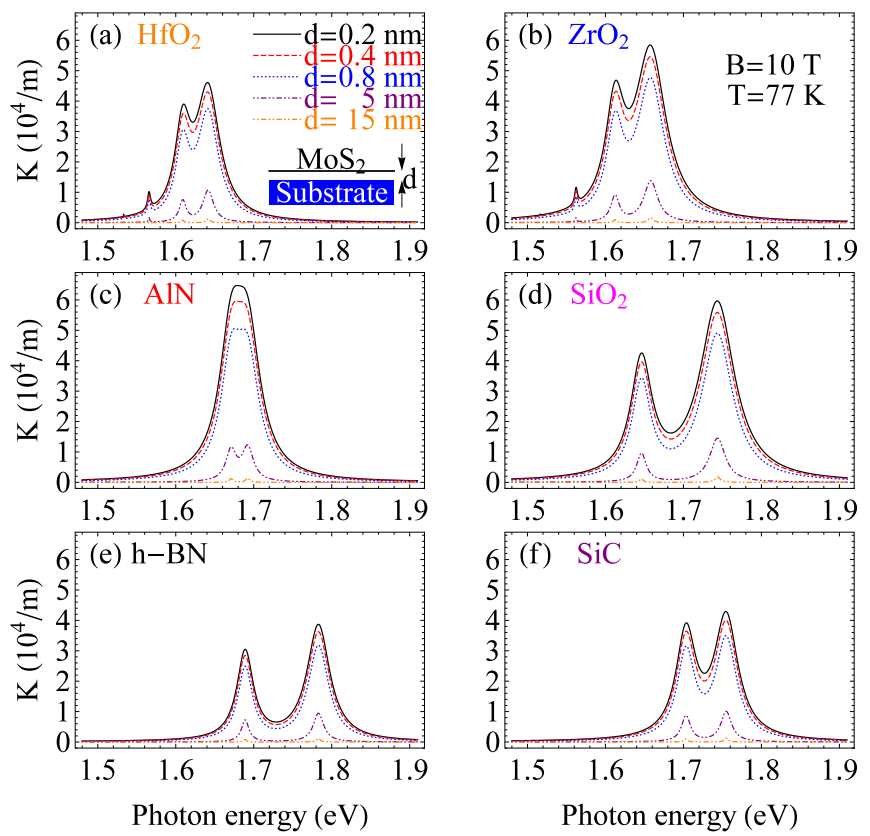

FIG. 17. Photon energy dependence of MOAC for $\mathrm{MoS}_{2}$ on several substrates due to electron-SO phonon interaction at different $\mathrm{MoS}_{2}$-substrate distance $d$. The results are calculated for spin up at $T=77 \mathrm{~K}$ and $B=10 \mathrm{~T}$.

the SO phonon mode from the substrate surface with the large distance [71,76,87]. As can be seen from Fig. 17, the MOAC is severely degraded when the effective $\mathrm{MoS}_{2}$-substrate distance is larger than $15 \mathrm{~nm}$. In this case the effect of SO phonon is very weak and can be neglected.

To further illustrate the effect of the distance $d$ on the absorption spectrum, in Fig. 18 we display the dependence of the FWHM on the distance $d$ for $\mathrm{MoS}_{2}$ on many different substrates at $B=10 \mathrm{~T}$ and $T=77 \mathrm{~K}$. The figure shows that the FWHM decreases nonlinearly with increasing distance $d$ in both one- and two-photon absorption processes as well as in

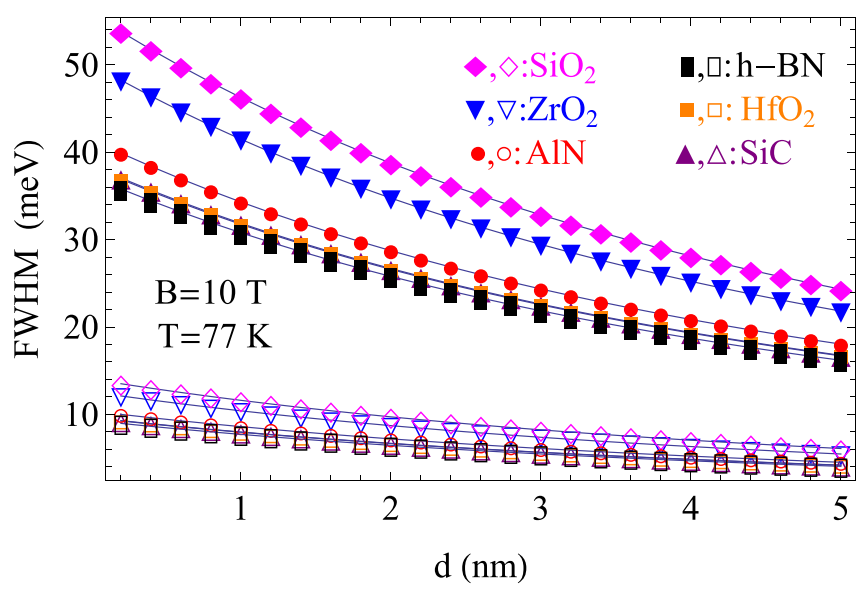

FIG. 18. $\mathrm{MoS}_{2}$-substrate distance dependence of FWHM for $\mathrm{MoS}_{2}$ on several substrates due to electron-SO phonon interaction. The results are calculated for spin up at $B=10 \mathrm{~T}$ and $T=77 \mathrm{~K}$. The full and empty dots correspond to the one- and two-photon absorption processes, respectively. all of the six substrates due to the decreasing of the probability of electron-SO phonon scattering. The FWHM is found to be largest for the case of an $\mathrm{SiO}_{2}$ substrate and smallest for h-BN resulting from the fact that electron-SO phonon scattering is strongest in $\mathrm{SiO}_{2}$ and weakest in h-BN.

\section{SUMMARY AND CONCLUSIONS}

Considering electron scattering from $\mathrm{MoS}_{2}$ intrinsic acoustic and optical phonons as well as the surface optical phonons induced by polar substrates, we studied the magneto-optical transport properties of monolayer $\mathrm{MoS}_{2}$ on several polar substrates subject to an external perpendicular magnetic field, including one- and two-photon absorption processes. Due to the large band gap of $\mathrm{MoS}_{2}$, the interband optical transitions are observed in the visible frequency range. The strong SOC is reflected in the splitting of the LLs leading to the separated energies of the spin up and spin down states.

The $\mathrm{MoS}_{2}$ intrinsic acoustic phonons have been investigated for both TA and LA modes and for both deformation potential and piezoelectric coupling mechanisms. Due to having the strongest ratio $D_{\lambda}^{\mu} / v_{s \lambda}$, TA phonons via PE coupling dominates MOAC and FWHM in comparison with other acoustic phonon scattering mechanisms while LA phonons via PE coupling shows the smallest. For the intrinsic optical phonon, the optical zero-order deformation potential and the Fröhlich interactions dominate MOAC and FWHM. Besides, SO phonons play an important role at high temperature depending on the substrate the $\mathrm{MoS}_{2}$ layer is placed upon. Our results show that the magnitude of the absorption peaks is strongly enhanced by substrates due to their strong electron-SO phonon coupling as compared to those for intrinsic acoustic and optical phonons. Moreover, because it has the strongest electron-SO phonon scattering, the $\mathrm{SiO}_{2}$ substrate is found to be dominant to MOAC and FWHM over the entire temperature and magnetic field range considered, while h-BN shows the smallest ones among the six substrates.

Furthermore, we have studied the influence of temperature, magnetic field, and the effective $\mathrm{MoS}_{2}$-substrate distance on MOAC and FWHM. With increasing temperature and magnetic field, both MOAC and FWHM are enhanced. Whereas, with increasing the $\mathrm{MoS}_{2}$-substrate distance these properties reduce rapidly. Besides, the $\mathrm{HfO}_{2}$ and $\mathrm{ZrO}_{2}$ substrates are strongly affected by temperature, especially for the absorbed SO phonon peaks, due to their large low-frequency dielectric constant and small SO phonon energies. The $\sqrt{B}$-dependent FWHM, except for the first order ODP case, is found to be in good agreement with that in the conventional $2 \mathrm{D}$ electron gas and that in graphene. We hope that this result would be useful for the orientation of experimental studies in monolayer $\mathrm{MoS}_{2}$ in the near future.

\section{ACKNOWLEDGMENTS}

This research is funded by the Vietnam National Foundation for Science and Technology Development (NAFOSTED) under Grant No. 103.01-2015.93 and the Belarusian scientific program "Convergence". 


\section{APPENDIX: MOAC VIA THE FIRST-ORDER ODP}

In the case of the first-order deformation potential interaction, the matrix element is given in the second part of Eq. (30). Similarly in the zero-order deformation potential interaction, by means of the dispersionless optical phonons $\omega_{\mathbf{q}, \lambda}=\omega_{\mathrm{TO}}$, the expression of the MOAC for inelastic scattering on a TO phonon is given as follows for the first-order deformation potential interaction:

$$
\begin{aligned}
K_{\mathrm{TO}}^{1}(\Omega)= & \mathcal{D}\left(\omega_{c}, \Omega\right) \frac{2 \hbar\left(D_{\mathrm{TO}}^{1}\right)^{2}}{\mathcal{A} \rho \omega_{\mathrm{TO}} a_{c}^{4}} \sum_{\alpha, \alpha^{\prime}}\left|\mathcal{B}_{\alpha \alpha^{\prime}}\right|^{2} f_{\alpha}\left(1-f_{\alpha^{\prime}}\right) \\
& \times \frac{\delta_{s s^{\prime}}}{L_{n, s}^{\eta, \tau} L_{n^{\prime}, s^{\prime}}^{\eta^{\prime} \tau}} N_{\mathrm{TO}}\left\{\mathcal{I}_{\alpha, \alpha^{\prime}}^{(1)}\left[\delta\left(\mathcal{Y}_{1}^{-}\right)+e^{\hbar \omega_{\mathrm{TO}} / k_{B} T} \delta\left(\mathcal{Y}_{1}^{+}\right)\right]\right. \\
& \left.+\mathcal{I}_{\alpha, \alpha^{\prime}}^{(2)}\left[\delta\left(\mathcal{Y}_{2}^{-}\right)+e^{\hbar \omega_{\mathrm{TO}} / k_{B} T} \delta\left(\mathcal{Y}_{2}^{+}\right)\right]\right\},
\end{aligned}
$$

where $N_{\text {TO }}$ is is the equilibrium Bose-Einstein distribution function for the phonons $\omega_{\mathrm{TO}}, \mathcal{I}_{\alpha, \alpha^{\prime}}^{(1)}=16 \mathcal{F}_{\alpha, \alpha^{\prime}}^{(2)} / \alpha_{0}^{2}, \mathcal{I}_{\alpha, \alpha^{\prime}}^{(2)}=$ $\left(\alpha_{0}^{2} / 16\right) \int_{0}^{\infty} q^{7}\left|J_{\alpha, \alpha^{\prime}}(q)\right|^{2} d q$, which will be numerically calculated, and $\mathcal{Y}_{\ell}^{ \pm}$is given the same form as $Y_{\ell}^{ \pm}$shown in Eq. (32) but with $\hbar \omega_{\mathrm{HP}}$ replaced by $\hbar \omega_{\mathrm{TO}}$. The delta functions in Eq. (A1) imply the energy conservation law, which shows the selection rule

$$
\ell \hbar \Omega=E_{\alpha^{\prime}}-E_{\alpha} \pm \hbar \omega_{\mathrm{TO}} .
$$

Finally, the corresponding Lorentzian widths for TO phonon scattering is given as follows:

$$
\left(\gamma_{\alpha, \alpha^{\prime}}^{ \pm, \mathrm{TO}}\right)^{2}=\frac{\hbar\left(D_{\mathrm{TO}}^{1}\right)^{2}}{2 \pi \rho \omega_{\mathrm{TO}} a_{c}^{4}} \frac{\delta_{s s^{\prime}}}{L_{n, s}^{\eta, \tau} L_{n^{\prime}, s^{\prime}}^{\eta^{\prime}, \tau}} \mathcal{F}_{\alpha, \alpha^{\prime}}^{(1)} N_{\mathrm{TO}}^{ \pm}
$$

Note that, unlike in the case of zero-order ODP where the Lorentzian widths are proportional to $a_{c}^{-1} \sim \sqrt{B}$ [see Eq. (34)], here we can see that the Lorentzian widths for TO phonon scattering are proportional to $a_{c}^{-2} \sim B$ which results in the linearly magnetic field-dependent FWHM as shown in Fig. 9.
[1] K. S. Novoselov, A. K. Geim, S. V. Morozov, D. Jiang, Y. Zhang, S. V. Dubonos, I. V. Grigorieva, and A. A. Firsov, Science 306, 666 (2004).

[2] A. K. Geim and K. S. Novoselov, Nat. Mater. 6, 183 (2007).

[3] K. S. Novoselov, V. I. Fal'ko, L. Colombo, P. R. Gellert, M. G. Schwab, and K. Kim, Nature (London) 490, 192 (2012).

[4] K. F. Mak, C. Lee, J. Hone, J. Shan, and T. F. Heinz, Phys. Rev. Lett. 105, 136805 (2010).

[5] B. Radisavljevic, A. Radenovic, J. Brivio, V. Giacometti, and A. Kis, Nat. Nanotechnol. 6, 147 (2011).

[6] K. F. Mak, K. He, J. Shan, and T. F. Heinz, Nat. Nanotechnol. 7, 494 (2012).

[7] K. Kaasbjerg, K. S. Thygesen, and K. W. Jacobsen, Phys. Rev. B 85, 115317 (2012).

[8] C. M. Wang and X. L. Lei, Phys. Rev. B 92, 125303 (2015).

[9] D. Xiao, G.-B. Liu, W. Feng, X. Xu, and W. Yao, Phys. Rev. Lett. 108, 196802 (2012).

[10] A. Scholz, T. Stauber, and J. Schliemann, Phys. Rev. B 88, 035135 (2013).

[11] M. Tahir, A. Manchon, and U. Schwingenschlögl, Phys. Rev. B 90, 125438 (2014).

[12] M. Tahir, P. Vasilopoulos, and F. M. Peeters, Phys. Rev. B 93, 035406 (2016).

[13] B. W. H. Baugher, H. O. H. Churchill, Y. Yang, and P. Jarillo-Herrero, Nano Lett. 13, 4212 (2013).

[14] D. Lembke and A. Kis, ACS Nano 6, 10070 (2012).

[15] A. Konar, T. Fang, and D. Jena, Phys. Rev. B 82, 115452 (2010).

[16] Y. J. Zhang, T. Oka, R. Suzuki, J. T. Ye, and Y. Iwasa, Science 344, 725 (2014).

[17] X. Xu, W. Yao, D. Xiao, and T. F. Heinz, Nat. Phys. 10, 343 (2014).

[18] K. F. Mak, K. L. McGill, J. Park, and P. L. McEuen, Science 344, 1489 (2014).

[19] H. Zeng, J. Dai, W. Yao, D. Xiao, and X. Cui, Nat. Nanotechnol. 7, 490 (2012).
[20] S. Wu, S. Buckley, J. R. Schaibley, L. Feng, J. Yan, D. G. Mandrus, F. Hatami, W. Yao, J. Vuckovic, A. Majumdar, and X. $\mathrm{Xu}$, Nature (London) 520, 69 (2015).

[21] L. F. Mattheiss, Phys. Rev. B 8, 3719 (1973).

[22] X. Li, F. Zhang, and Q. Niu, Phys. Rev. Lett. 110, 066803 (2013).

[23] A. Kormányos, P. Rakyta, and G. Burkard, New J. Phys. 17, 103006 (2015).

[24] C. V. Nguyen, N. N. Hieu, and V. V. Ilyasov, J. Electron. Mater 45, 4038 (2016).

[25] C. V. Nguyen and N. N. Hieu, Chem. Phys. 468, 9 (2016).

[26] P. Lu, X. Wu, W. Guo, and X. C. Zeng, Phys. Chem. Chem. Phys. 14, 13035 (2012).

[27] Z. Li and J. P. Carbotte, Phys. Rev. B 86, 205425 (2012).

[28] J. He, K. Wu, R. Sa, Q. Li, and Y. Wei, Appl. Phys. Lett. 96, 082504 (2010).

[29] F. Rose, M. O. Goerbig, and F. Piéchon, Phys. Rev. B 88, 125438 (2013).

[30] X. Zhou, Y. Liu, M. Zhou, D. Tang, and G. Zhou, J. Phys.: Condens. Matter 26, 485008 (2014).

[31] P. M. Krstajić, P. Vasilopoulos, and M. Tahir, Phys. Rev. B 94 085413 (2016).

[32] Y. Ge, W. Wan, W. Feng, D. Xiao, and Y. Yao, Phys. Rev. B 90, 035414 (2014).

[33] W. Li, J. Carrete, and N. Mingo, Appl. Phys. Lett. 103, 253103 (2013).

[34] Z. Li and J. P. Carbotte, Phys. B 421, 97 (2013).

[35] Z. Li and J. P. Carbotte, Phys. Rev. B 88, 045417 (2013).

[36] Z. Li and J. P. Carbotte, Eur. Phys. J. B 88, 87 (2015).

[37] K. Kaasbjerg, K. S. Thygesen, and A.-P. Jauho, Phys. Rev. B 87, 235312 (2013).

[38] K. Kaasbjerg, K. S. Bhargavi, and S. S. Kubakaddi, Phys. Rev. B 90, 165436 (2014).

[39] K. S. Bhargavi, S. Patil, and S. S. Kubakaddi, J. Appl. Phys. 118, 044308 (2015).

[40] K. Ghosh and U. Singisetti, J. Appl. Phys. 118, 135711 (2015). 
[41] X. Li, J. T. Mullen, Z. Jin, K. M. Borysenko, M. Buongiorno Nardelli, and K. W. Kim, Phys. Rev. B 87, 115418 (2013).

[42] Z.-W. Wang, R.-Z. Li, Y. Xiao, and Z.-Q. Li, Phys. Lett. A 380, 3843 (2016).

[43] B. J. Robinson, C. E. Giusca, Y. T. Gonzalez, N. D. Kay, O. Kazakova, and O. V. Kolosov, 2D Mater. 2, 015005 (2015).

[44] B. Scharf, V. Perebeinos, J. Fabian, and I. Žutić, Phys. Rev. B 88, 125429 (2013).

[45] H. V. Phuc and L. Dinh, Mater. Chem. Phys. 163, 116 (2015).

[46] A. Mogulkoc, Y. Mogulkoc, A. N. Rudenko, and M. I. Katsnelson, Phys. Rev. B 93, 085417 (2016).

[47] X. Zhou, W.-K. Lou, F. Zhai, and K. Chang, Phys. Rev. B 92, 165405 (2015).

[48] Z. Li and J. P. Carbotte, Phys. Rev. B 88, 045414 (2013).

[49] M. Lasia and L. Brey, Phys. Rev. B 90, 075417 (2014).

[50] B. Fallahazad, H. C. P. Movva, K. Kim, S. Larentis, T. Taniguchi, K. Watanabe, S. K. Banerjee, and E. Tutuc, Phys. Rev. Lett. 116, 086601 (2016).

[51] P. E. C. Ashby and J. P. Carbotte, Phys. Rev. B 87, 245131 (2013).

[52] C. J. Tabert and E. J. Nicol, Phys. Rev. Lett. 110, 197402 (2013).

[53] C. J. Tabert and E. J. Nicol, Phys. Rev. B 88, 085434 (2013).

[54] X. Y. Zhou, R. Zhang, J. P. Sun, Y. L. Zou, D. Zhang, W. K. Lou, F. Cheng, G. H. Zhou, F. Zhai, and K. Chang, Sci. Rep. 5, 12295 (2015).

[55] M. Tahir, P. Vasilopoulos, and F. M. Peeters, Phys. Rev. B 92 , 045420 (2015).

[56] M. Tahir and P. Vasilopoulos, Phys. Rev. B 94, 045415 (2016).

[57] R.-L. Chu, X. Li, S. Wu, Q. Niu, W. Yao, X. Xu, and C. Zhang, Phys. Rev. B 90, 045427 (2014).

[58] W.-Y. Shan, H.-Z. Lu, and D. Xiao, Phys. Rev. B 88, 125301 (2013).

[59] V. P. Gusynin, S. G. Sharapov, and J. P. Carbotte, Phys. Rev. Lett. 98, 157402 (2007).

[60] M. Koshino and T. Ando, Phys. Rev. B 77, 115313 (2008).

[61] S. L. Chuang, Physics of Optoelectronic Devices (Wiley, New York, 1995).

[62] H. V. Phuc and N. N. Hieu, Opt. Commun. 344, 12 (2015).

[63] W. Xu, R. A. Lewis, P. M. Koenraad, and C. J. G. M. Langerak, J. Phys.: Condens. Matter 16, 89 (2004).

[64] W. Xu, Phys. Rev. B 57, 12939 (1998).
[65] K. Seeger, Semiconductor Physics: An Introduction (Springer, Berlin, 1985), Vol. 40.

[66] W.-K. Tse and A. H. MacDonald, Phys. Rev. B 84, 205327 (2011).

[67] N. Ma and D. Jena, Phys. Rev. X 4, 011043 (2014).

[68] P. Vasilopoulos, M. Charbonneau, and C. M. Van Vliet, Phys. Rev. B 35, 1334 (1987).

[69] C. H. Yang, F. M. Peeters, and W. Xu, Phys. Rev. B 82, 075401 (2010).

[70] Z. Y. Zhu, Y. C. Cheng, and U. Schwingenschlögl, Phys. Rev. B 84, 153402 (2011).

[71] S. Fratini and F. Guinea, Phys. Rev. B 77, 195415 (2008).

[72] C. M. Wang and X. L. Lei, Phys. Rev. B 87, 235403 (2013).

[73] L. Zeng, Z. Xin, S. Chen, G. Du, J. Kang, and X. Liu, Appl. Phys. Lett. 103, 113505 (2013).

[74] B. Scharf, V. Perebeinos, J. Fabian, and P. Avouris, Phys. Rev. B 87, 035414 (2013).

[75] J. Sak, Phys. Rev. B 6, 3981 (1972).

[76] S. Q. Wang and G. D. Mahan, Phys. Rev. B 6, 4517 (1972).

[77] N. Mori and T. Ando, Phys. Rev. B 40, 6175 (1989).

[78] X. Cui, G.-H. Lee, Y. D. Kim, G. Arefe, P. Y. Huang, C.-H. Lee, D. A. Chenet, X. Zhang, L. Wang, F. Ye, F. Pizzocchero, B. S. Jessen, K. Watanabe, T. Taniguchi, D. A. Muller, T. Low, P. Kim, and J. Hone, Nat. Nanotechnol. 10, 534 (2015).

[79] H. V. Phuc, N. N. Hieu, L. Dinh, and T. C. Phong, Opt. Commun. 335, 37 (2015).

[80] N. Shon and T. Ando, J. Phys. Soc. Jpn. 67, 2421 (1998).

[81] M. E. Raikh and T. V. Shahbazyan, Phys. Rev. B 47, 1522 (1993).

[82] Y. Zheng and T. Ando, Phys. Rev. B 65, 245420 (2002).

[83] Z. Jiang, E. A. Henriksen, L. C. Tung, Y.-J. Wang, M. E. Schwartz, M. Y. Han, P. Kim, and H. L. Stormer, Phys. Rev. Lett. 98, 197403 (2007).

[84] H. V. Phuc, Superlattices Microstruct. 88, 518 (2015).

[85] M. Orlita, C. Faugeras, P. Plochocka, P. Neugebauer, G. Martinez, D. K. Maude, A.-L. Barra, M. Sprinkle, C. Berger, W. A. de Heer, and M. Potemski, Phys. Rev. Lett. 101, 267601 (2008).

[86] I.-T. Lin and J.-M. Liu, Appl. Phys. Lett. 103, 081606 (2013).

[87] J.-H. Chen, C. Jang, S. Xiao, M. Ishigami, and M. S. Fuhrer, Nat. Nanotechnol. 3, 206 (2008). 